\title{
DENSE CORES IN GALAXIES OUT TO $z=2.5$ IN SDSS, UltraVISTA, AND THE FIVE 3D-HST/CANDELS FIELDS
}

\author{
Pieter G. van Dokkum ${ }^{1}$, Rachel Bezanson ${ }^{2}$, ArJen van der Wel $^{3}$, Erica June Nelson ${ }^{1}$, Ivelina Momcheva $^{1}$, \\ Rosalind E. Skelton ${ }^{4}$, Katherine E. Whitaker ${ }^{5}$, Gabriel Brammer ${ }^{6}$, Charlie Conroy $^{7}$, \\ Natascha M. Förster Schreiber ${ }^{8}$, Mattia Fumagalli ${ }^{9}$, Mariska Kriek $^{10}$, Ivo Labbé ${ }^{9}$, Joel Leja ${ }^{1}$, \\ Danilo Marchesini $^{11}$, Adam Muzzin ${ }^{9}$, Pascal Oesch $^{1}$, and Stijn Wuyts ${ }^{9}$ \\ ${ }^{1}$ Department of Astronomy, Yale University, New Haven, CT 06511, USA; pieter.vandokkum@ yale.edu \\ ${ }^{2}$ Steward Observatory, University of Arizona, 933 North Cherry Avenue, Tucson, AZ 85721, USA \\ ${ }^{3}$ Max Planck Institute for Astronomy (MPIA), Königstuhl 17, D-69117 Heidelberg, Germany \\ ${ }^{4}$ South African Astronomical Observatory, PO Box 9, Observatory, Cape Town 7935, South Africa \\ ${ }^{5}$ Astrophysics Science Division, Goddard Space Center, Greenbelt, MD 20771, USA \\ ${ }^{6}$ Space Telescope Science Institute, Baltimore, MD 21218, USA \\ ${ }^{7}$ Department of Astronomy and Astrophysics, University of California, Santa Cruz, CA 95064, USA \\ ${ }^{8}$ Max-Planck-Institut für Extraterrestrische Physik, Giessenbachstrasse, D-85748 Garching, Germany \\ ${ }^{9}$ Leiden Observatory, Leiden University, 2300-RA Leiden, The Netherlands \\ ${ }^{10}$ Department of Astronomy, University of California, Berkeley, CA 94720, USA \\ ${ }^{11}$ Department of Physics and Astronomy, Tufts University, Medford, MA 02155, USA \\ Received 2014 March 19; accepted 2014 June 30; published 2014 July 24
}

\begin{abstract}
The dense interiors of massive galaxies are among the most intriguing environments in the universe. In this paper,we ask when these dense cores were formed and determine how galaxies gradually assembled around them. We select galaxies that have a stellar mass $>3 \times 10^{10} M_{\odot}$ inside $r=1 \mathrm{kpc}$ out to $z=2.5$, using the 3D-HST survey and data at low redshift. Remarkably, the number density of galaxies with dense cores appears to have decreased from $z=2.5$ to the present. This decrease is probably mostly due to stellar mass loss and the resulting adiabatic expansion, with some contribution from merging. We infer that dense cores were mostly formed at $z>2.5$, consistent with their largely quiescent stellar populations. While the cores appear to form early, the galaxies in which they reside show strong evolution: their total masses increase by a factor of $2-3$ from $z=2.5$ to $z=0$ and their effective radii increase by a factor of 5-6. As a result, the contribution of dense cores to the total mass of the galaxies in which they reside decreases from $\sim 50 \%$ at $z=2.5$ to $\sim 15 \%$ at $z=0$. Because of their early formation, the contribution of dense cores to the total stellar mass budget of the universe is a strong function of redshift. The stars in cores with $M_{1 \mathrm{kpc}}>3 \times 10^{10} M_{\odot}$ make up $\sim 0.1 \%$ of the stellar mass density of the universe today but $10 \%-20 \%$ at $z \sim 2$, depending on their initial mass function. The formation of these cores required the conversion of $\sim 10^{11} M_{\odot}$ of gas into stars within $\sim 1 \mathrm{kpc}$, while preventing significant star formation at larger radii.
\end{abstract}

Key words: cosmology: observations - galaxies: evolution - Galaxy: formation - Galaxy: structure

Online-only material: color figures

\section{INTRODUCTION}

The central regions of massive elliptical galaxies such as NGC 1399 and NGC 4472 are different from any environment seen in galaxies such as the Milky Way. The mean stellar densities are $\sim 10 M_{\odot} \mathrm{pc}^{-3}$ in the central kpc, and their velocity dispersions reach or even exceed $\sim 300 \mathrm{~km} \mathrm{~s}^{-1}$. The stellar populations are old, metal-rich, and strongly $\alpha$-enhanced, indicating that the stars were formed early in a short, intense period of star formation (Franx \& Illingworth 1990; Worthey et al. 1992; Davies et al. 1993; Kuntschner et al. 2001, 2010, and many other studies). Star formation in these central regions likely took place under very different physical conditions than those in the present-day disk of the Milky Way, possibly leading to a bottom-heavy initial mass function (IMF) with an excess of low mass stars compared to the Milky Way IMF (van Dokkum \& Conroy 2010; Treu et al. 2010; Krumholz 2011; Cappellari et al. 2012; Conroy \& van Dokkum 2012; Hopkins 2013). These dense centers also host the most massive black holes in the universe (Magorrian et al. 1998; Ferrarese \& Merritt 2000; Gebhardt et al. 2000), which probably accreted most of their mass during the peak star formation epoch. Despite their high star formation efficiency in the past, dense regions are hostile to star formation today: quiescence correlates well with velocity dispersion and with stellar surface density (Kauffmann et al. 2003; Franx et al. 2008; Wake et al. 2012; Bell et al. 2012).

The dense interiors of massive galaxies account for only a small fraction of the total stellar mass in the present-day universe, but given the old ages of their stars this fraction is expected to increase with redshift. In fact, the formation of the dense central parts of elliptical galaxies may precede the assembly of the rest of the galaxies. Many quiescent galaxies at $z=1.5-2.5$ are much more compact than nearby galaxies of the same mass (e.g., Daddi et al. 2005; Trujillo et al. 2006; van Dokkum et al. 2008; Cimatti et al. 2008; Damjanov et al. 2009; Williams et al. 2010), and as first shown by Bezanson et al. (2009) the central densities of the compact high redshift galaxies are broadly similar to those of massive elliptical galaxies today. This is consistent with the idea that massive galaxies have grown inside-out since $z \sim 2$, with their cores forming at higher redshift and their outer envelopes building up slowly through star formation, minor mergers, or other processes (e.g., Loeb \& Peebles 2003; Bezanson et al. 2009; Naab et al. 2009; van Dokkum et al. 2010; Hopkins et al. 2010; Oser et al. 2010; Feldmann et al. 2010; Szomoru et al. 2013).

In this paper, we focus exclusively on these dense central regions of massive galaxies: we ask what their number density is, what their contribution is to the overall stellar mass density, 
and how the galaxies that they are part of were built up around them. In practice, we select galaxies out to $z=2.5$ that have $\log M_{1 \mathrm{kpc}} \gtrsim 10.5$, that is, a stellar mass exceeding $3.2 \times 10^{10} M_{\odot}$ within a sphere of radius $r=1 \mathrm{kpc} .{ }^{12}$ We do not limit the sample to quiescent galaxies but select all objects that satisfy this stellar density criterion. Our approach is different from studies of the properties of galaxies at fixed total stellar mass, or fixed number density. In fact, as we show in Section 4, the evolution of the number density of galaxies with $\log M_{1 \mathrm{kpc}}>10.5$ is different from that of the general population of massive galaxies. Our study is more closely related to the work of Bezanson et al. (2011) on the evolution of the velocity dispersion function; Bezanson et al. converted observed effective radii, stellar masses, and Sersic (1968) indices to velocity dispersions whereas we convert the same parameters to a stellar mass within a physical radius of $1 \mathrm{kpc}$.

In this paper, we do not make any a priori selection on star formation rate or galaxy size. Nevertheless, this paper has implications for the evolution of massive quiescent galaxies at $z \sim 2$. It is generally thought that these galaxies have grown substantially in size over the past $10 \mathrm{Gyr}$, but this interpretation is complicated by the fact that the number density of quiescent galaxies has increased by an order of magnitude over this time period (Brammer et al. 2011; Cassata et al. 2013; Muzzin et al. 2013a). As discussed by van Dokkum et al. (2008), van der Wel et al. (2009), Trujillo et al. (2011), Newman et al. (2012), Carollo et al. (2013), Szomoru et al. (2013), and others, the evolution of the mass-size relation of quiescent galaxies could be partially driven by the continuous addition of large, recently quenched star-forming galaxies, in which case the growth of individual quiescent galaxies would be smaller than that of the population. Some studies have even suggested that compact quiescent galaxies barely evolve at all (e.g., Poggianti et al. 2013). As we show in Section 4, the evolution of galaxies with dense cores appears to require substantial evolution in the sizes and masses of individual compact galaxies after $z \sim 2$.

The paper is structured as follows. In Section 2, we describe the sources of data that are used. In Section 3, the selection of galaxies with dense cores is described. Sections 4 and 5 form the heart of the paper. In Section 4, the "core mass function" is discussed, that is, the number of galaxies as a function of their mass within $1 \mathrm{kpc}$. This section also presents the evolution of the cumulative number density of galaxies with $\log M_{1 \mathrm{kpc}}>10.5$, and interprets the evolution in the context of various physical processes. Finally, it places the total stellar mass locked up in dense cores in the context of the evolving stellar mass density of the universe. In Section 5, the properties of galaxies that have dense cores are analyzed; here we show that the core-hosting galaxies likely evolved significantly since $z \sim 2$, increasing both their total mass and (particularly) their effective radii. We also discuss the nature of star-forming galaxies with dense cores. The paper is summarized in Section 6.

\section{DATA}

\subsection{The 3D-HST Survey and Catalog}

We use the imaging, spectroscopy, and catalogs from the 3D-HST survey (Brammer et al. 2012). 3D-HST is an Hubble Space Telescope (HST) Treasury program that has provided

\footnotetext{
12 We refer to the region within this radius as the "core" throughout this paper, realizing that this may cause confusion. The same term has been used

extensively in the literature to describe the surface density profile of early-type galaxies on much smaller scales (e.g., Faber et al. 1997).
}

WFC3/G141 grism spectroscopy over four of the five extragalactic fields imaged by the CANDELS survey (Grogin et al. 2011; Koekemoer et al. 2011). Including archival data on GOODS-North from program GO-11600 (PI: Weiner) approximately $80 \%$ of the CANDELS area is covered by grism observations.

In addition to analyzing the grism spectroscopy, the 3D-HST project reduced all the CANDELS WFC3 imaging, and has constructed photometric catalogs in the five CANDELS fields using publicly available ground- and space-based photometry from $0.3 \mu \mathrm{m}$ to $8.0 \mu \mathrm{m}$. This multi-wavelength photometry aids in the interpretation of the grism spectroscopy and is obviously valuable in its own right. The complete CANDELS + 3D-HST data sets in all five CANDELS fields are included in v4.1 of the catalogs. ${ }^{13}$ Here we give a brief description of the 3D-HST data products; the full catalogs are presented and described in Skelton et al. (2014).

Redshifts were measured from a combination of the $U$-IRAC photometric data and the WFC3/G141 grism spectra, using a modified version of the EAZY code (Brammer et al. 2008) as described in Brammer et al. (2013). Comparisons to ground-based spectroscopic redshifts suggest an accuracy of 0.003-0.005 in $\Delta z /(1+z)$ for galaxies with $H \lesssim 23$; this seems to be borne out by stacking analyses of galaxies without a previously measured redshift (see Whitaker et al. 2013). For faint galaxies, and in areas of the CANDELS fields that do not have grism coverage, we use the photometric redshift instead. Structural parameters in $J_{125}$ and $H_{160}$ were measured using GALFIT (Peng et al. 2002), as described in van der Wel et al. (2014). The Sersic (1968) parameters measured from the 3D-HST mosaics are in excellent agreement with those measured from the CANDELS mosaics (van der Wel et al. 2012) for the same objects. Stellar masses were measured from the photometric data using the FAST code (Kriek et al. 2009b), assuming a Chabrier (2003) IMF.

Excluding the areas surrounding bright stars and regions with little WFC3 exposure time (such as the edge of the field), the five fields cover a total of $896 \mathrm{arcmin}^{2}$ in version 4.1 of our catalogs: $192.4 \operatorname{arcmin}^{2}$ in AEGIS, $183.9 \operatorname{arcmin}^{2}$ in COSMOS, $157.8 \mathrm{arcmin}^{2}$ in GOODS-North, $171.0 \mathrm{arcmin}^{2}$ in GOODSSouth, and $191.2 \mathrm{arcmin}^{2}$ in the Ultra Deep Survey (UDS) field (see Grogin et al. 2011; Skelton et al. 2014, for a detailed description of these fields).

\subsection{The UltraVISTA and "Zürich" Catalogs in the COSMOS Field}

Dense cores are rare and large volumes are required to measure their number density accurately. As we show in Section 4, the number density of galaxies with $\log \left(M_{1 \mathrm{kpc}}\right)>$ 10.5 is less than $10^{-4} \mathrm{Mpc}^{-3}$ at low redshift. The volume probed by the 3D-HST/CANDELS survey is only $1.7 \times 10^{5} \mathrm{Mpc}^{3}$ at $0<z<0.5$, which means of order 10 galaxies can be expected. As this redshift range covers approximately half of the time elapsed since $z=2.5$, we augment the 3D-HST/CANDELS survey with the wide-field UltraVISTA survey in the COSMOS field.

We use the deep $K$-selected catalog of Muzzin et al. (2013b), which is based on the data described in McCracken et al. (2012). The data sets and procedures used by Muzzin et al. are similar to those used in our analysis of the CANDELS/3D-HST data. In particular, photometric redshifts and stellar masses were derived using the same software and assumptions, which means they

\footnotetext{
$\overline{13}$ http://3dhst.research.yale.edu/Home.html
} 
can be combined with our higher redshift data. This catalog was matched to the "Zürich Structure and Morphology" catalog v1.0 (Sargent et al. 2007), which contains structural parameters of objects in the COSMOS field to a limiting magnitude of $I=22.5$. Sizes and Sersic indices were derived from the $H S T /$ ACS $I_{814}$ imaging in this field (Scoville et al. 2007), using the GIM2D software (Simard et al. 2002). The total area that is covered by both UltraVISTA and Advanced Camera for Surveys (ACS) is $1.54 \mathrm{deg}^{2}$, a factor of six larger than the 3D-HST/ CANDELS survey. We note that the COSMOS objects studied in this paper, galaxies with dense cores at $0<z<0.5$, are bright and far removed from the limits of the data. Also, the $I_{814}$ images are well matched in rest-frame wavelength to the $J_{125}$ and $H_{160}$ that are used at higher redshifts.

\subsection{The Sloan Digital Sky Survey}

We also make use of a local sample, drawn from the 7 th data release of the Sloan Digital Sky Survey (SDSS). This sample is described in Bezanson et al. (2013); it is based on structural parameters measured by Simard et al. (2011) and $M / L$ ratios from the MPA-JHU catalog (Brinchmann et al. 2004). The following cuts were applied: keep_flag $=1, z_{-}$warning $=0$, sciencePrimary $=1$, and a requirement that structural parameters and masses are available from Simard et al. (2011) and the MPA-JHU catalog, respectively. ${ }^{14}$ An effective area of $8032 \mathrm{deg}^{2}$ was assumed (see Simard et al. 2011). Unfortunately, we have no direct test to assess whether the SDSS masses and sizes are on the same system as the 3D-HST data. There is no evidence to the contrary: as shown in Bezanson et al. (2013) there are no obvious systematic differences in sizes or masses between this SDSS sample and the extrapolation to $z=0$ of distant galaxy samples analyzed in the same way as done here.

\section{CORE MASSES}

\subsection{Selection at $0.5<z<2.5$}

The parent sample constitutes all galaxies in the five 3D-HST/CANDELS fields that have a photometric "use" flag of 1 (see Skelton et al. 2014) and a structural parameter fit flag of 0 or 1 (see van der Wel et al. 2012, 2014). For all these galaxies (essentially the entire 3D-HST v4.0 catalog), we calculated the stellar mass that is contained within a radius of $1 \mathrm{kpc}$. Following Bezanson et al. (2009), we first deprojected the best-fitting Sersic profile using an Abel transform:

$$
\rho(x)=\frac{b_{n}}{\pi} \frac{I_{0}}{r_{e}} x^{1 / n-1} \int_{1}^{\infty} \frac{\exp \left(-b_{n} x^{1 / n} t\right)}{\sqrt{t^{2 n}-1}} d t,
$$

with $\rho$ the three-dimensional (3D) luminosity density in a particular filter, $x \equiv r / r_{e}, r_{e}$ the circularized effective radius, $n$ the Sersic index, and $b_{n}$ the $n$-dependent normalization parameter of the Sersic profile (see, e.g., Peng et al. 2002). This deprojection is important as the projected mass within $1 \mathrm{kpc}$ is influenced by the properties of the galaxy at larger radius. In particular, for a given density (in $M_{\odot} \mathrm{kpc}^{-3}$ ) within a sphere of radius $1 \mathrm{kpc}$, larger and more massive galaxies have a higher projected density (in $M_{\odot} \mathrm{kpc}^{-2}$ ). We note that this methodology may lead to errors for galaxies that are far from spherical symmetry, in particular for flat disks. We return to this in Section 5.3.

\footnotetext{
14 Bezanson et al. (2013) also required the error in the measured stellar velocity dispersion to be below $10 \%$. This additional constraint was not applied here.
}

Next, the mass within $r=1 \mathrm{kpc}$ was calculated by integrating the 3D luminosity profiles. A small (typically $<10 \%$ ) correction to the masses was applied to take into account that the total magnitude in the catalog is not identical to the total magnitude implied by the Sersic fit (see Taylor et al. 2010a). This same correction was applied to the total masses used in later sections. We also assume that mass follows light. This assumption is probably reasonable in a relative sense, as there is no evidence that color gradients (and hence $M / L$ gradients) are a strong function of redshift (see Szomoru et al. 2013). Combining all these aspects, the core mass is given by

$$
M_{1 \mathrm{kpc}}=\frac{\int_{0}^{1 \mathrm{kpc}} \rho(r) r^{2} d r}{\int_{0}^{\infty} \rho(r) r^{2} d r} \frac{L_{\text {model }}}{L_{\text {cat }}} M_{\text {cat }} .
$$

Here, $M_{\text {cat }}$ is the mass of the galaxy in the 3D-HST catalog, $L_{\text {cat }}$ is the total, aperture-corrected luminosity of the galaxy in a particular filter in the $3 \mathrm{D}-\mathrm{HST}$ catalog, and $L_{\text {model }}$ is the total luminosity implied by the Sersic fit.

Finally, the measurements for $M_{1 \mathrm{kpc}}$ derived from the $J_{125}$ fit and from the $H_{160}$ fit were interpolated so that the final value corresponds to a rest-frame wavelength of $6000 \AA$ for $z=1.0-1.7$. At $z<1$ we use $J_{125}$ and at $z>1.7$ we use $H_{160}$. We note that this interpolation is not a critical step: the effect on the derived masses is typically $<5 \%$, and using the core mass derived from either $J_{125}$ or $H_{160}$ throughout does not change the results significantly.

The difference between total mass and core mass is illustrated in Figure 1. The figure shows $H_{160}$ images of three galaxies at $z \sim 1$, along with a graphical representation of their surface density. The galaxy on the left is a massive elliptical galaxy with a dense core. Not all massive galaxies have a high central density: the middle galaxy has a mass that is nearly identical to the galaxy on the left and a relatively low mass core. The galaxy on the right is an example of a galaxy whose total mass is only slightly higher than its core mass; this galaxy resembles the compact quiescent galaxies that are relatively common at high redshift. The galaxies on the left and right are included in our $\log M_{1 \mathrm{kpc}}>10.5$ sample (see Section 4), and the galaxy in the middle is not.

We note here that we do not measure the (deprojected) light in the central regions directly, but use the best-fitting Sersic profiles as a proxy for the light at $r<1 \mathrm{kpc}$. The central kpc covers $\sim 12$ drizzled WFC3 pixels, and we could have used a direct measurement of the flux within this aperture. Using the Sersic fits instead takes the effects of the point-spread function into account, and enables the deprojection described above. As described in van der Wel et al. $(2012,2014)$ these fits are stable even for galaxies with $r_{e} \sim 1 \mathrm{kpc}$; the total uncertainties in $n$ and $r_{e}$ are $<20 \%$ and $<10 \%$, respectively, for $H_{160}<23$ (see van der Wel et al. 2012). However, it is possible that our results are affected by (large) galaxies whose surface brightness profiles deviate strongly from a Sersic profile.

\subsection{Low Redshift Samples}

The procedures followed for the UltraVISTA/Zürich galaxies at $0<z<0.5$ and for the SDSS galaxies at $z \approx 0.06$ were similar to those described above. The only difference in procedure is that the UltraVISTA masses were not corrected for the difference between total catalog magnitudes and the total fluxes implied by the GIM2D fit. In Appendix A, we compare the UltraVISTA masses to the 3D-HST masses for objects that are in both catalogs. A redshift-dependent offset 


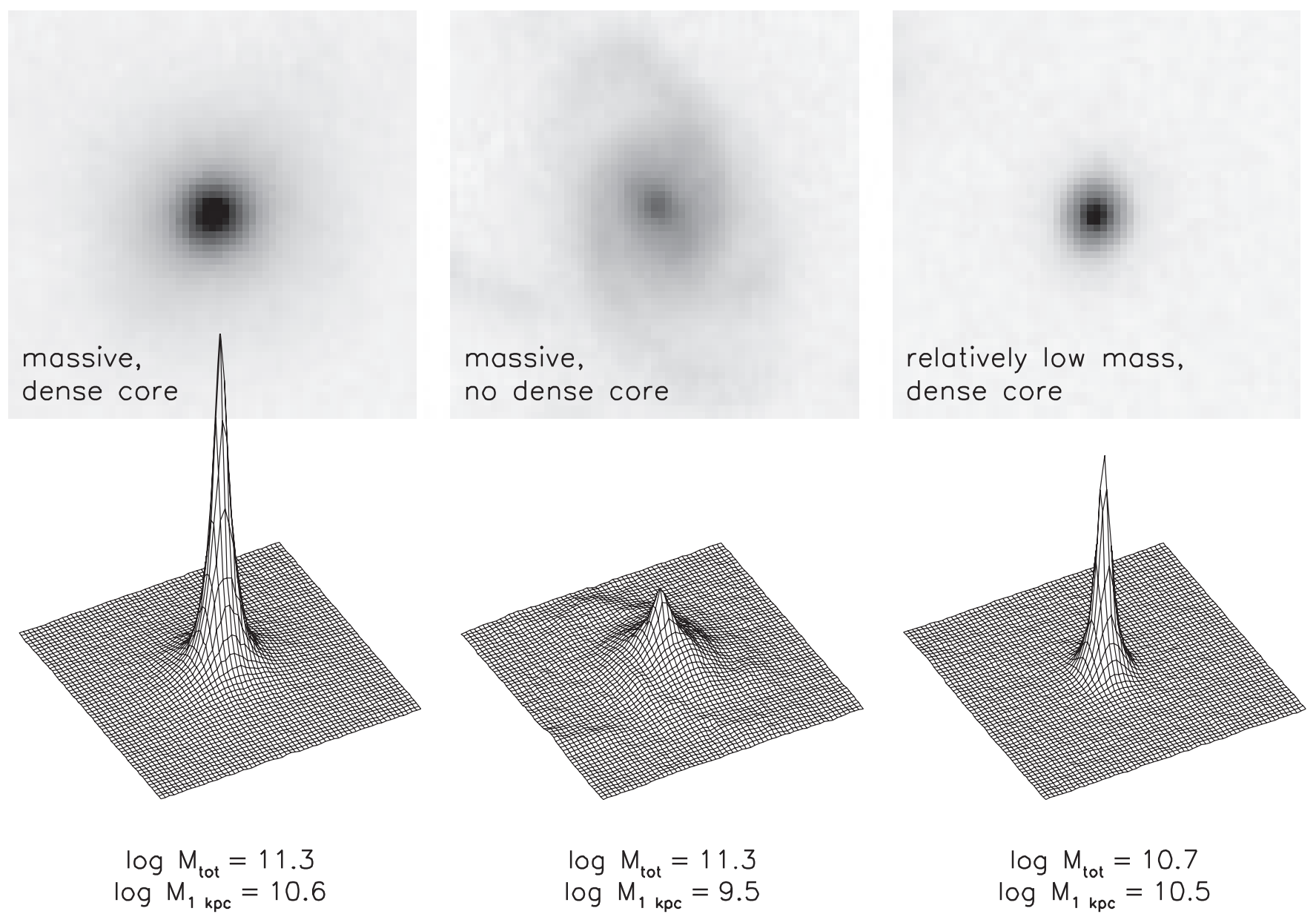

Figure 1. Illustration of the distinction between central mass and total mass. The panels show HST/WFC3 $H_{160}$ images of three galaxies at $z \sim 1$; each panel spans $30 \mathrm{kpc} \times 30 \mathrm{kpc}$. The galaxy on the left has a high total mass and a high core mass; the middle galaxy has a high total mass but a low core mass; and the galaxy on the right is compact with a relatively low total mass and a high core mass.

was applied to the UltraVISTA masses so they are consistent with the 3D-HST masses. The origin of this offset is not understood; we conservatively increase the formal uncertainty in the UltraVISTA masses by the same amount so that an offset of zero, or of twice the applied offset, are within the $1 \sigma$ error bars in all plots. After applying this offset the core masses derived from UltraVISTA/Zürich are consistent with those derived from $3 \mathrm{D}-\mathrm{HST}$ to $0.00 \pm 0.02 \mathrm{dex}$ (see Appendix A).

\subsection{Relation Between Total Mass and Core Mass}

In Figure 2, we show the relation between the total mass of galaxies and the mass within $1 \mathrm{kpc}$ in six redshift bins from $z=0$ to $z=2.5$. All galaxies are shown; there was no selection on star formation rate or any other property. The 3D-HST catalog is $90 \%-95 \%$ complete for masses $>10^{10} M_{\odot}$ out to $z=2.5$ (van der Wel et al. 2014). ${ }^{15}$ It is clear from this figure that the central mass is not a fixed fraction of the total mass: the $68 \%$ range in $\log \left(M_{\mathrm{tot}}\right)$ at fixed $\log \left(M_{1 \mathrm{kpc}}\right)$ is approximately 0.5 dex. A selection on core mass is therefore distinct from a selection on total mass (see also, e.g., Kauffmann et al. 2003; Franx et al. 2008).

Starting with the SDSS sample and going to higher redshifts, the distribution of points shifts and tilts such that at fixed total mass galaxies have higher core masses at higher redshifts. The

\footnotetext{
15 The completeness gradually decreases at higher redshifts, partially because the $4000 \AA$ break enters the observed WFC $3 H_{160}$ filter.
}

dashed line shows the line of equality: objects near this line are so compact that they have close to $100 \%$ of their mass in the central kpc. At low redshift nearly all galaxies are far removed from this regime, particularly at the high mass end. However, at $z>1.5$ the distribution begins to approach this line, with the most pronounced change at the highest masses. The galaxies are color-coded by their projected circularized effective radius. As is well known, massive galaxies with small sizes are extremely rare in the nearby universe (Taylor et al. 2010b; Trujillo et al. 2009). Mirroring the trend between core mass and total mass, and consistent with many previous studies (e.g., Daddi et al. 2005; van Dokkum et al. 2008), we see that such galaxies are increasingly common at higher redshifts.

\section{NUMBER DENSITIES}

\subsection{The "Core Mass Function"}

In the remainder of the paper, we select objects using the $x$ axis of Figure 2 and ask what the properties are of galaxies at fixed core mass rather than fixed total mass. We first consider the number density of galaxies as a function of their mass within $1 \mathrm{kpc}$, the "core mass function." This function is shown in Figure 3 for the same six redshift intervals as in Figure 2. For reference, the SDSS core mass function (panel (a)) is shown in panels (b)-(f) with a dashed line. 


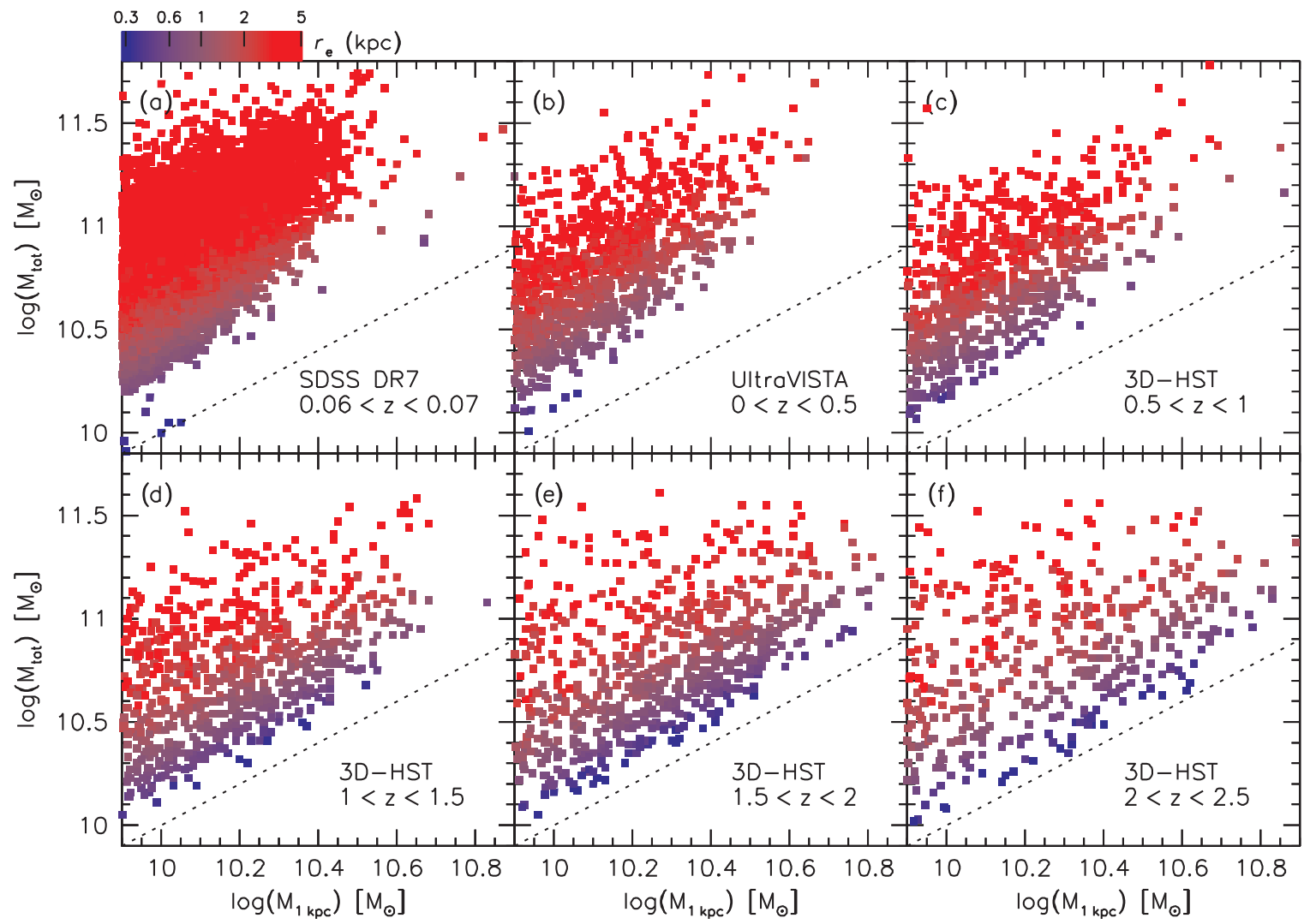

Figure 2. Relation between total stellar mass and the stellar mass in a sphere of radius $1 \mathrm{kpc}$. The panels show (a) nearby galaxies from the Sloan Digital Sky Survey, (b) galaxies at moderate redshift from the UltraVISTA and "Zürich" surveys of the $1.5 \mathrm{deg}^{2}$ COSMOS field, and ((c)-(f)) distant galaxies from the 3D-HST survey of the CANDELS fields. The galaxies are color-coded by their projected, circularized effective radius. At fixed total mass, galaxies have progressively smaller sizes and higher core masses at higher redshifts.

(A color version of this figure is available in the online journal.)

The core mass function evolves with redshift in a complex way. Going from low to high redshift, low mass cores decrease strongly in number density: the number of cores with $\log \left(M_{1 \mathrm{kpc}}\right)=10$ is a factor of $\sim 6$ lower at $z=2-2.5$ than it is at $z=0$. By contrast, the number of high mass cores apparently increases with redshift. The high mass end of the $z=2-2.5$ core mass function is shifted by $\approx 0.2 \mathrm{dex}$ in mass compared to that at $z=0.06$. Because of the steepness of the mass function, the number of cores with $\log \left(M_{1 \mathrm{kpc}}\right)=10.7$ increases by more than an order of magnitude from $z=0$ to $z=2.5$. In Appendix B, we show that random errors do not significantly influence this result.

This evolution is similar to the evolution of the (inferred) velocity dispersion function (Bezanson et al. 2011): this function shows a similar differential evolution of high dispersion and low dispersion galaxies, at least from $z=0.3$ to $z=1.5$. This is obviously not a completely independent measurement: as the velocity dispersions and central masses are both, to first order, measures of the compactness of galaxies they are expected to trace one another.

\subsection{Evolution of the Cumulative Number Density of Massive Cores}

We show the evolution of the number density of galaxies with a central mass $\log \left(M_{1 \mathrm{kpc}}\right)>10.5$ in Figure 4. The error bars reflect the quadratic sum of the Poisson error and a systematic error, calculated by varying the masses of the galaxies by \pm 0.05 dex in $3 \mathrm{D}$-HST, \pm 0.09 dex in UltraVISTA, and $\pm 0.1 \mathrm{dex}$ in SDSS. The SDSS error reflects the systematic uncertainty compared to the 3D-HST masses; in this paper the 3D-HST "system" is taken as the default. The value of 0.09 dex is the offset that was applied to bring the UltraVISTA data onto the 3D-HST system (see Appendix A). The evolution can be approximated by the solid line, which has the form

$$
\log (\Phi)=(-4.9 \pm 0.2)+(1.5 \pm 0.5) \log (1+z)
$$

This increase in the number density of dense cores with redshift is remarkable, as it is well established that the mass density of the universe decreases rapidly over this same redshift range (e.g., Dickinson et al. 2003; Rudnick et al. 2003). Although massive galaxies evolve less rapidly than the overall population (Marchesini et al. 2009), even their number density does not increase with redshift. We explicitly compare the evolution of dense cores to the evolution of massive galaxies with $\log \left(M_{\text {tot }}\right)>11$ in Figure 4, showing both our data (open circles) and number densities of massive galaxies derived from the NEWFIRM Medium Band Survey (NMBS) by Brammer et al. (2011; open squares). At $z \sim 2$, the number density of massive cores is only about a factor of two lower than that of massive galaxies. At $z \sim 0$, the number density is a factor of 100 lower. The striking difference in the evolution of massive galaxies and massive cores in Figure 4 strongly suggests that Equation (3) is not driven by mass errors, as errors in the masses should affect the open circles in the same way as the solid circles. In Appendix C, we also show that field-to-field variation ("cosmic variance") is not dominating the error budget. 


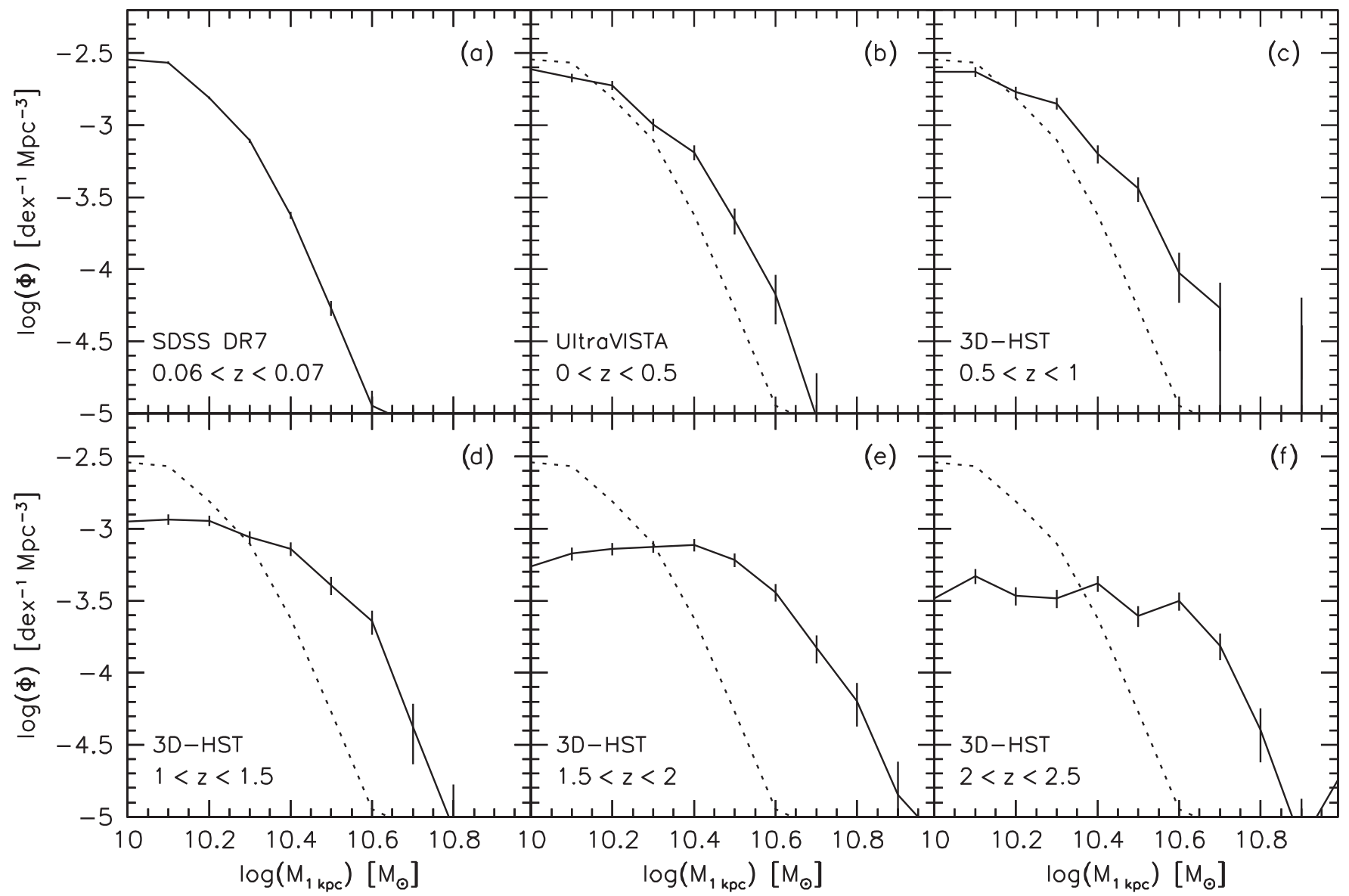

Figure 3. Evolution of the core mass function, i.e., the number of galaxies as a function of their mass within 1 kpc. Panels show (a) data from the SDSS, (b) UltraVISTA/Zürich, and ((c)-(f)) 3D-HST. The SDSS function is repeated in the other panels (broken lines). The shape of the core mass function changes with redshift: the number of low mass cores decreases with redshift, whereas the number of high mass cores increases.

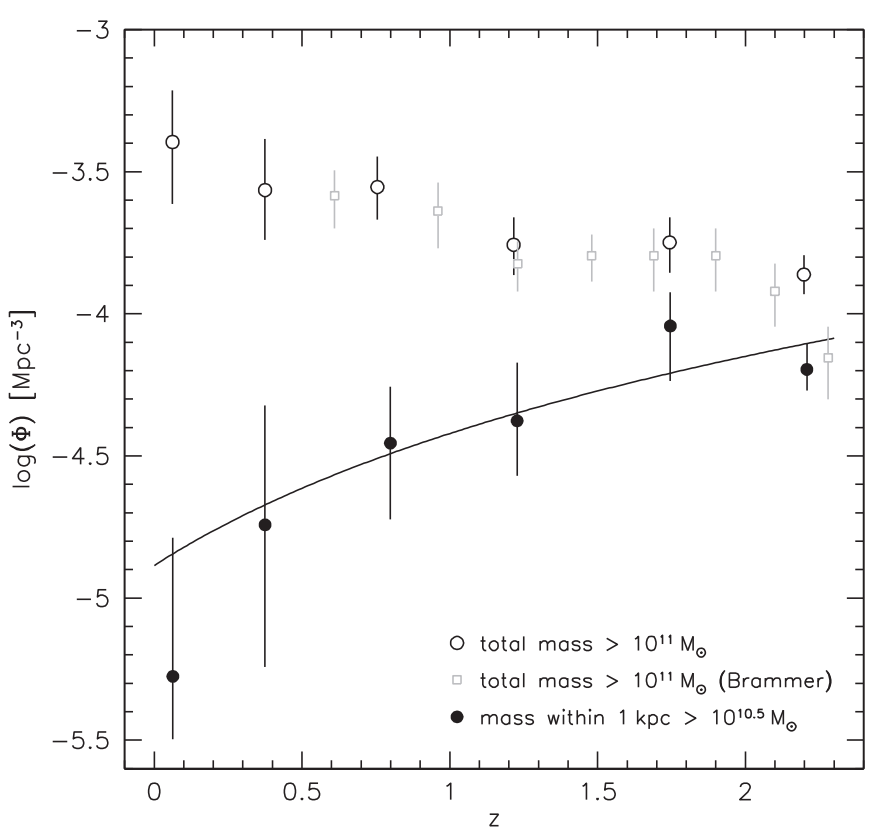

Figure 4. Evolution of the number density of galaxies that have dense cores with $\log \left(M_{1 \mathrm{kpc}}\right)>10.5$ (solid black circles). The number of dense cores was higher in the past. The line is a fit to the data. Open symbols show the evolution of massive galaxies with $\log \left(M_{\text {tot }}\right)>11$, from this paper (black circles) and from Brammer et al. (2011; gray squares). The number density of massive galaxies decreases with redshift, and evolves in a very different way than the number density of dense cores.
We note that there are indications of a similar effect in previous studies. The evolution of the velocity dispersion function from $z=0.3$ to $z=1.5$ is consistent with our results (Bezanson et al. 2011), and van de Sande et al. (2013) find that, at constant dynamical mass, the central $(<1 \mathrm{kpc})$ stellar density of quiescent galaxies at $z \sim 2$ is a factor of $\sim 3$ higher than at $z=0$.

\subsection{Effects of Mergers on the Number Density}

Setting aside the possibility of errors in the masses or the structural parameters, there are several plausible explanations for the observed evolution in Figure 4. We first consider the effects of mergers. Major mergers can increase the core mass, either through dissipationless processes (e.g., Hilz et al. 2013) or through merger-induced star formation (Solomon et al. 1992; Kormendy \& Sanders 1992; Hopkins et al. 2008, and many other studies). The most straightforward way to decrease the number density of galaxies with $\log \left(M_{1 \mathrm{kpc}}\right)>10.5$ is if two galaxies with such dense cores merge with each other.

We determined the number of potential core-core mergers in the 3D-HST survey, using the same criteria as Williams et al. (2011) to identify paired galaxies. In the entire 3D-HST/ CANDELS area we find three galaxy pairs with a projected distance $d<43 \mathrm{kpc}$, a redshift difference $\left|z_{1}-z_{2}\right| /\left(1+z_{1}\right)<$ 0.2 , and $\log \left(M_{1 \mathrm{kpc}}\right)>10.5$ for both galaxies. The pairs are at $z \approx 1.7, z \approx 2.0$, and $z \approx 2.3$; images are shown in Appendix D. The individual galaxies are well separated; no tidal features are detected. Interestingly all three pairs are red and in apparent 
overdensities of other red objects; we will return to this in a future paper.

The pair fraction is defined as the number of pairs divided by the number of galaxies in the parent population. The total number of galaxies with $\log \left(M_{1 \mathrm{kpc}}\right)>10.5$ is 273 , and we infer that the pair fraction is $1.1 \% \pm 0.6 \%$. This is a factor of $\sim$ five lower than the pair fraction in the general population of massive galaxies (Bell et al. 2006; Bundy et al. 2009; Williams et al. 2011). Specifically, Williams et al. (2011) find a pair fraction of $6 \% \pm 1 \%$ when requiring that the paired galaxies have masses that are within a factor of four of one another and the most massive galaxy has $\log M_{\text {tot }}>10.5$.

Turning pair fractions into merger rates is notoriously difficult, as it depends on the fraction of pairs that is physically bound and the average time it takes for bound pairs to merge (e.g., Bell et al. 2006; Kitzbichler \& White 2008). Nevertheless, it is clear that a pair fraction of $1 \%$ implies a low core-core merger rate. The separation of the three pairs is $\sim 30 \mathrm{kpc}$. Taking the orbital time as a lower limit on the merger time scale and assuming $v \sim 500 \mathrm{~km} \mathrm{~s}^{-1}$, we find that the pairs may merge after 200 Myr. The actual merger time scale (including a correction for unbound pairs) is probably significantly larger (see Kitzbichler \& White 2008). Using a pair fraction of $1 \%$, we derive an upper limit on the merger rate of $\sim 5 \%$ per Gyr. We note that if we use a distance limit of $100 \mathrm{kpc}$ rather than $43 \mathrm{kpc}$ we find a higher pair fraction (of $\sim 2 \%$ ) but a similar merger rate, as the orbital time is longer for pairs with wider separation. We infer that the decline in the number density of galaxies with massive cores is probably not caused by major mergers between galaxies with dense cores.

As the direct effect of core-core merging on the number density is probably small, the observed evolution of the core mass function is most likely a reflection of mass evolution in the central kpc (or of systematic errors). As can be seen in Figure 3, mass evolution of -0.15 to -0.2 dex is sufficient to bring the low redshift and high redshift core mass functions into agreement. This mass evolution may be the result of a redistribution of matter following a merger (e.g., El-Zant et al. 2004; Oser et al. 2012). Mergers are thought to be common, particularly at redshifts $0<z<1.5$ (e.g., van Dokkum 2005; Naab et al. 2009; Newman et al. 2012; Bluck et al. 2012, and Section 5). The central regions of galaxies are thought to be mostly unaffected by minor mergers (see, e.g., Figure 3 in Hilz et al. 2013). This is also suggested by the results of Weinmann et al. (2013), who have shown that the number density of dark matter halos with fixed high central density is constant to within $\sim 0.2$ dex at $0<z<4$. We stress, however, that the quantitative effects of mergers on the mass profile within $1 \mathrm{kpc}$ are not well known.

\subsection{Effects of Stellar Winds}

As is well known "negative" mass evolution is expected (and inevitable) in an isolated stellar population: the mass locked up in stars and stellar remnants decreases with time due to supernova explosions and stellar winds. The amount of mass loss is a strong function of the mass and surface gravity of the stars that are present in the population, and therefore a strong function of both the age and the IMF.

Previous studies have considered the effects of mass loss on the evolution of the mass-size relation, particularly in the context of the size growth of quiescent galaxies (Damjanov et al. 2009). As mass loss leads to adiabatic expansion, passively evolving galaxies become both less massive and larger with time; however, these effects are small compared to the observed evolution of the sizes and masses of quiescent galaxies (Damjanov et al. 2009; Bezanson et al. 2009; Ragone-Figueroa \& Granato 2011).

Here we are concerned with a slightly different question, namely the effect on the mass contained within a sphere of $1 \mathrm{kpc}$. The mass within a fixed radius is affected by stellar mass loss in two ways. The first, direct, effect is that the stellar mass measurements of galaxies at later times will be lower, as they only include living stars and stellar remnants and not the mass that is lost during stellar evolution. The second effect is that the matter in galaxies can be redistributed as a result of adiabatic expansion. This effect is only important if the material in the winds mixes with the hot halo gas, which in turn depends on the details of the interaction of the wind material with the ambient gas (e.g., Bregman \& Parriott 2009; Conroy et al. 2014). We show in Appendix E that the total effect of stellar mass loss is

$$
\left(M^{\prime} / M\right)_{1 \mathrm{kpc}} \sim\left(M^{\prime} / M\right)_{\mathrm{tot}}^{1.8},
$$

with $\left(M^{\prime} / M\right)_{\text {tot }}$ the change in the total mass and $\left(M^{\prime} / M\right)_{1 \mathrm{kpc}}$ the change in mass in the central kpc. The relation in Equation (4) assumes that all the material escapes and there are no other sources of mass loss than stellar evolution. If no material escapes, Equation (4) is simply $\left(M^{\prime} / M\right)_{1 \mathrm{kpc}} \sim\left(M^{\prime} / M_{\mathrm{tot}}\right.$.

The change in the total mass due to stellar evolution can be estimated using stellar population synthesis models. The main uncertainty is the age of the stars, with the added complexity that the ages in the central kpc can be different from the ages at larger radii. We consider two mean formation redshifts of the stars, $z_{*}=2.5$ and $z_{*}=5$, and two IMFs, a Milky Waylike Kroupa (2001) IMF and a bottom-heavy Salpeter (1955) IMF. The top panel of Figure 5 shows the mass evolution of a passively evolving stellar population for the four model combinations, determined using the Bruzual \& Charlot (2003) stellar population synthesis model. At late times the mass loss is approximately $30 \%$ for a Salpeter IMF and $50 \%$ for a Kroupa IMF. The relative mass loss from $z=2.5$ to $z=0$ depends on the combination of the IMF and the formation redshift of the stars. In Figure 5(b) we show the effect on the mass within $1 \mathrm{kpc}$, relative to $z=2.2$ (our highest redshift bin). The minimum effect of mass loss is $\approx 0.06 \mathrm{dex}$ (for $z_{*}=5$ and a bottom-heavy $\mathrm{IMF}$ ) and the maximum effect is $\approx 0.22 \mathrm{dex}$ (for $z_{*}=2.5$ and a bottom-light IMF).

In Figure 5(c), we show the effect of mass loss on the evolution of the number density of dense cores. We adapted the selection of galaxies, so that we select cores of lower mass at lower redshift. This redshift dependence is given by the relation $\Delta \log \left(M_{1 \mathrm{kpc}}\right)=0.06 \times z($ solid black line in Figure 5(b)), which is a linear fit to the mass loss expected for a Salpeter (1955) IMF with $z_{*}=2.5$. This is also a good fit to the mass loss for a Kroupa (2001) IMF with $z_{*}=5$, but it is lower than the mass loss expected for a Kroupa (2001) IMF with $z_{*}=2.5$ (see Figure 5(b)). The solid points in Figure 5(c) show the number density of galaxies that have $\log \left(M_{1 \mathrm{kpc}}\right)>10.5+0.06 \times(z-2.2)$. The error bars reflect the systematic uncertainties in the masses, as before. We find that the data are now consistent with a constant number density since $z=2.5$, within the uncertainties. The weighted mean number density is $\Phi \approx 7 \times 10^{-5} \mathrm{Mpc}^{-3}$.

We conclude that the mass evolution in the central $\mathrm{kpc}$ can be explained with a passively evolving population formed at $z>2.5$, under the assumption that $100 \%$ of the mass lost during stellar evolution mixes with the hot halo gas. In reality, the central mass decrease probably reflects a combination of 


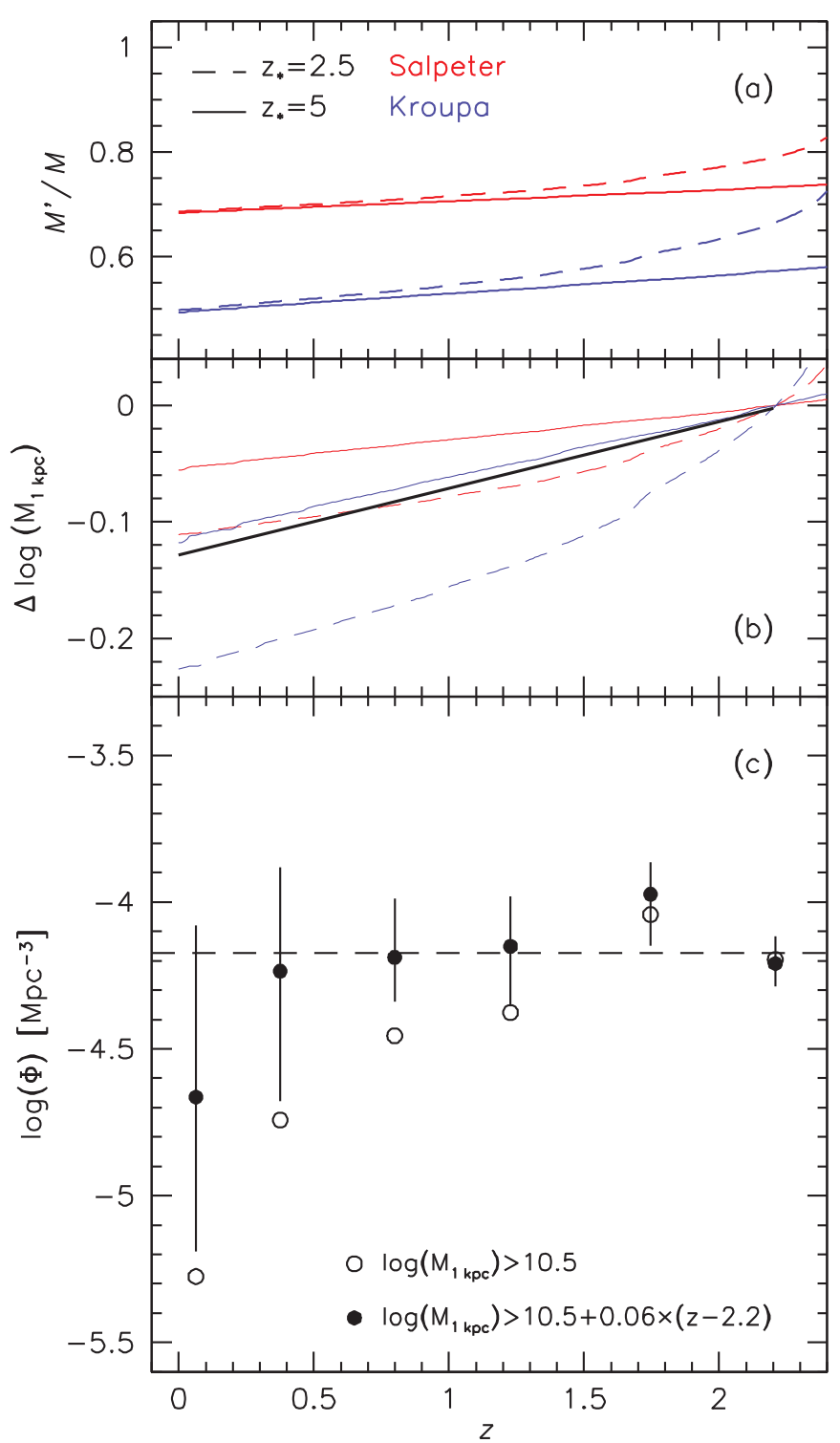

Figure 5. (a) Stellar mass evolution of a passively evolving stellar population. The mass decreases with time, mostly due to winds from asymptotic giant branch (AGB) and post-AGB stars. Mass loss is largest for young stellar populations and bottom-light IMFs. (b) Evolution of the mass within $1 \mathrm{kpc}$. The evolution is stronger than in panel (a) due to the effects of adiabatic expansion, under the assumption that the stellar ejecta turbulently mix with the ambient hot gas. The black line is a linear fit to the $z_{*}=2.5$ Salpeter model, of the form $\Delta \log \left(M_{1 \mathrm{kpc}}\right)=0.06 z$. (c) The number density of dense cores, after correcting the core masses for stellar mass loss according to the black line in panel (b). The data are consistent with a constant number density of $\sim 7 \times 10^{-5} \mathrm{Mpc}^{-3}$ (dashed line).

(A color version of this figure is available in the online journal.)

the effects of mergers and mass loss, and it will be difficult to disentangle these effects.

\subsection{Other Explanations}

We briefly consider several other explanations for the apparent negative evolution of the number density of dense cores. One possibility is that the structure of galaxies changes in such a way that the deprojected core masses change systematically with redshift. Specifically, in the deprojection we use the circularized effective radius and if the mean flattening of galaxies changes with redshift this may lead to artificial offsets between the high redshift and low redshift data. In Appendix F we show the core mass function derived using the major axis effective radius rather than the circularized effective radius. The function is offset to lower masses, as expected, but there is no significant redshift-dependent effect.

Another interesting possibility is that the mass in the cores decreases due to scouring by binary supermassive black holes. The presence of flat density profiles in the centers of massive, slowly rotating elliptical galaxies ${ }^{16}$ (Faber et al. 1997) has been attributed to the ejection of stars by a binary black hole (see, e.g., Begelman et al. 1980; Milosavljević \& Merritt 2001; Kormendy \& Bender 2009; Hopkins \& Hernquist 2010). The total amount of mass that is ejected is of order $M_{\text {ej }} \sim M_{\bullet}$ (e.g., Milosavljević \& Merritt 2001). Our adopted core mass limit of $3 \times 10^{10} M_{\odot}$ within $1 \mathrm{kpc}$ corresponds to a velocity dispersion of $\sigma \sim 280 \mathrm{~km} \mathrm{~s}^{-1}$, which in turn corresponds to a black hole mass of $M_{\bullet} \sim 5 \times 10^{8} M_{\odot}$ (Gültekin et al. 2009). The ratio of the black hole mass to the stellar mass within $1 \mathrm{kpc}$ is therefore $\sim 2 \%$. Even taking adiabatic expansion into account, we infer that the stellar mass within $1 \mathrm{kpc}$ is only reduced by 0.01-0.02 dex due to black hole scouring, unless $M_{\text {ej }} \gg M_{\bullet}$ (see Hopkins \& Hernquist 2010 for a recent discussion on this topic).

Perhaps the most important alternative explanation is systematic error. A redshift-dependent error in the stellar masses of 0.05-0.1 dex per unit redshift could fully explain the evolution. Combined with the (inevitable) effects of mass loss, the (small) effects of black hole scouring, and the (uncertain) effects of merging, we conclude that the uncertainties allow a factor of $\sim$ two evolution in the number density, in either direction.

\subsection{Contribution of Stars in Dense Cores to the Stellar Mass Density of the Universe}

As discussed in the Introduction, the dense cores studied in this paper are extreme environments by local universe standards. They contain only a small fraction of the total stellar mass in the universe, and as we showed in Figure 4 the number density of galaxies with $\log M_{1 \mathrm{kpc}}>10.5$ is a factor of $\sim 100$ lower than that of galaxies with $\log M_{\text {tot }}>11$.

However, the fact that their number density and mass does not increase with time, and probably even decreases, means dense cores are an increasingly important environment at higher redshift. Going back in time from the present to $z \sim 2.5$, we see galaxies such as the Milky Way "lose" 90\% of their stars (van Dokkum et al. 2013) whereas the dense cores become ever more prominent and striking: their mass increases and the galaxies in which they are embedded today are stripped away (see Section 5).

Figure 6(a) shows the integrated stellar mass in cores of mass $\log \left(M_{1 \mathrm{kpc}}\right)>10.5$ as a function of redshift. This is not the total stellar mass of all galaxies hosting such cores: only stars within the central $1 \mathrm{kpc}$ are counted. The stellar mass density rises steeply with redshift, reflecting the increase in the number density shown in Figure 4. Figure 6(a) also shows the stellar mass density of the universe, taken from Table 2 of Muzzin et al. (2013a). Muzzin et al. calculated these mass densities by integrating the best-fitting Schechter functions down to $10^{8} M_{\odot}$. We added 0.1 dex in quadrature to the uncertainties listed in Muzzin et al. (2013a) to account for possible systematic differences between 3D-HST and UltraVISTA (see Appendix A). The stellar mass density of the universe decreases with redshift, and the

\footnotetext{
16 Also termed "cores"; see footnote 12.
} 

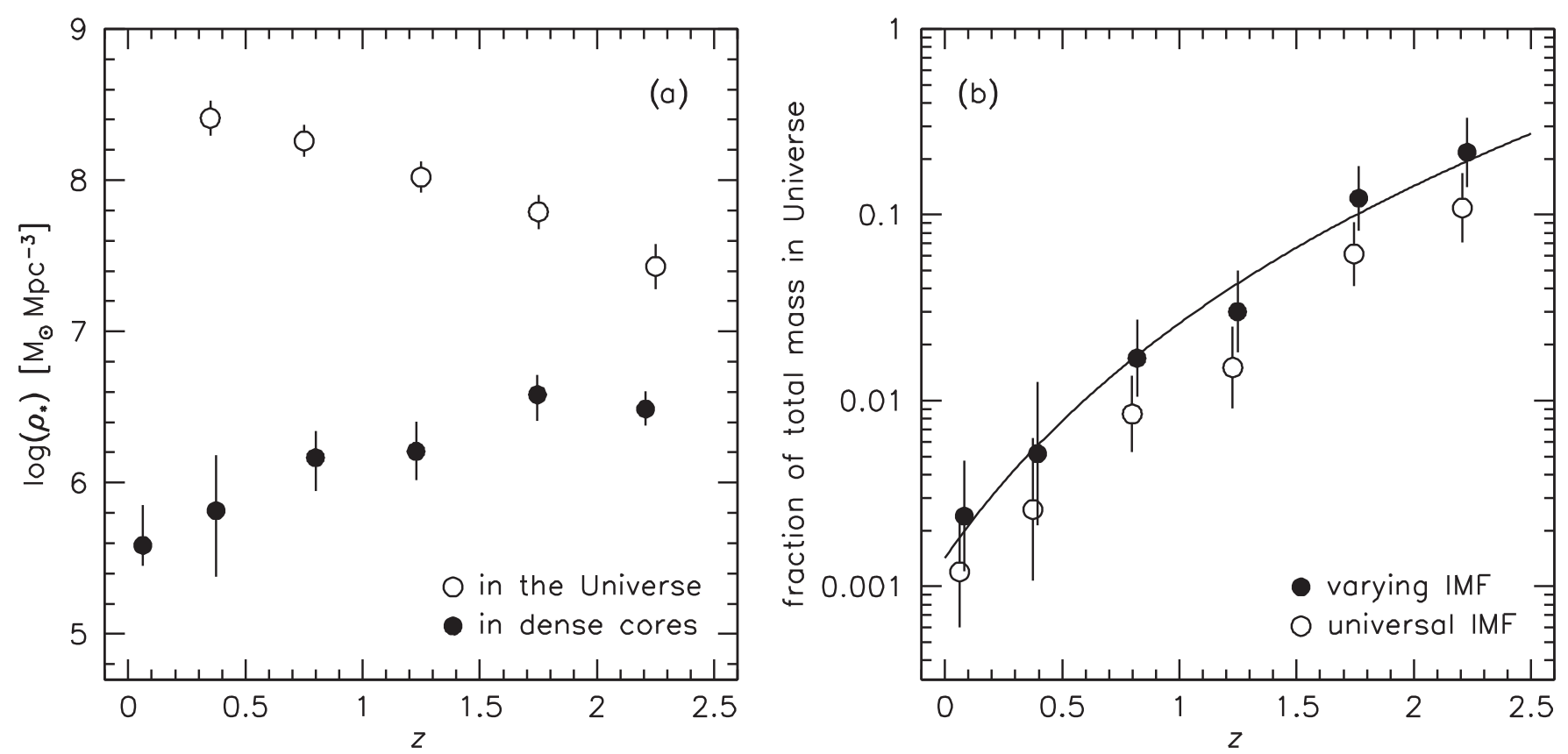

Figure 6. (a) Total stellar mass contained in cores with $\log \left(M_{1 \mathrm{kpc}}\right)>10.5$, as a function of redshift (solid circles). Open circles show the total stellar mass density of the universe, as determined by Muzzin et al. (2013a). A Kroupa (2001) IMF was assumed, for the cores and for the universe. (b) Fraction of the total stellar mass density of the universe that is contained in cores with $\log \left(M_{1 \mathrm{kpc}}\right)>10.5$. Open symbols assume a universal IMF, solid symbols (slightly offset for clarity) assume that the stellar mass function in dense cores has a factor of two more mass than that in the rest of the universe, as might be expected if the IMF in star-forming cores was bottom-heavy (Conroy \& van Dokkum 2012). Stars in dense cores comprise 10\%-20\% of the stellar mass density at $z>2$, and reflect an important mode of starand galaxy formation in the early universe.

stars living in dense cores make up a rapidly increasing fraction of the total stellar mass density.

In Figure 6(b) we show this fraction explicitly. It rises from $\sim 0.1 \%$ at $z=0$ to $\sim 10 \%$ at $z=2$. The fraction is even higher if the IMF is bottom heavy in dense regions, as has been inferred from studies of absorption lines and the masses of nearby galaxies (e.g., van Dokkum \& Conroy 2010; Treu et al. 2010; Cappellari et al. 2012; Conroy \& van Dokkum 2012). Somewhat dependent on the detailed functional form of the IMF, Conroy \& van Dokkum (2012) find that galaxies with high velocity dispersions have stellar masses that are a factor of $\sim$ two higher than would be inferred from a Kroupa (2001) IMF. The solid points in Figure 6(b) indicate the effect of such IMF variation. The line is a fit to the solid points of the form

$$
f=1.4 \times 10^{-3} \times(1+z)^{4.2},
$$

with $f$ the fraction of the total stellar mass density in the universe that is locked up in dense cores. The implication is that the stars in dense cores may constitute $\sim 20 \%$ of the total stellar mass density at $z=2.2$. Phrased differently, $\sim 20 \%$ of all the stars formed at $z \gtrsim 2.5$ may have ended up in dense cores.

\section{BUILD-UP OF GALAXIES AROUND DENSE CORES}

\subsection{Evolution of Total Mass and Effective Radius}

We now turn to the properties of the galaxies in which the dense cores reside. The fact that the number density of galaxies with dense cores is relatively stable with time has an important implication. It means that we can plausibly identify the descendants of high redshift galaxies with dense cores. In the general population of massive galaxies the number density at fixed mass increases with time, and as different galaxies can have different growth rates there is an inherent uncertainty in linking progenitors and descendants (e.g., Newman et al. 2012; Leja et al. 2013). In contrast, galaxies with dense cores are consistent with having a passive stellar population in their central $1 \mathrm{kpc}$, enabling, in principal, a one-to-one matching of galaxies across cosmic time.

In practice processes such as mergers, stellar winds, occasional star formation, and black hole scouring make this comparison less straightforward (see Sections 4.3-4.5). Here we take mass loss due to stellar winds into account but ignore all other effects, including possible systematic errors. We use the black solid line in Figure 5(b) to parameterize mass loss:

$$
\log \left(M_{1 \mathrm{kpc}}^{\text {evo }}\right)\left[M_{\odot}\right]=10.37+0.06 z .
$$

As discussed in Section 4.4, this parameterization is a linear fit to the mass loss expected for a Salpeter (1955) IMF with $z_{*}=2.5$. We measure the build-up of massive galaxies around their dense cores by selecting galaxies in a narrow mass bin centered on this evolving mass.

Figure 7 (left) shows the total masses of galaxies with a core mass $\log \left(M_{1 \mathrm{kpc}}\right)=\log \left(M_{1 \mathrm{kpc}}^{\text {evo }}\right) \pm 0.05$. The total masses increase with time, despite the fact that we selected the galaxies to have a central mass that decreases with time. The total mass evolves as

$$
\log \left(M_{\mathrm{tot}}\right)\left[M_{\odot}\right]=(11.21 \pm 0.04)-(0.70 \pm 0.08) \log (1+z)
$$

with the errors determined from bootstrap resampling. This fit is indicated by the solid line in Figure 7 (left). The evolution is slightly faster if we correct for stellar mass loss. As $\left(M^{\prime} / M\right)_{\mathrm{tot}} \sim\left(M^{\prime} / M_{1 \mathrm{kpc}}\right)^{0.6}$ this correction $\Delta \log \left(M_{\mathrm{tot}}\right) \approx$ $0.6 \times 0.06 z \approx 0.04 z$. The mass evolution is then $\log \left(M_{\text {tot,cor }}\right) \approx$ $11.30-0.87 \log (1+z)$. Going to higher redshifts, dense cores make up an increasing fraction of the total mass of the galaxies that they are part of: at $z=0$ their contribution is $\approx 15 \%$ and at $z=2.5$ it is $\approx 50 \%$. 

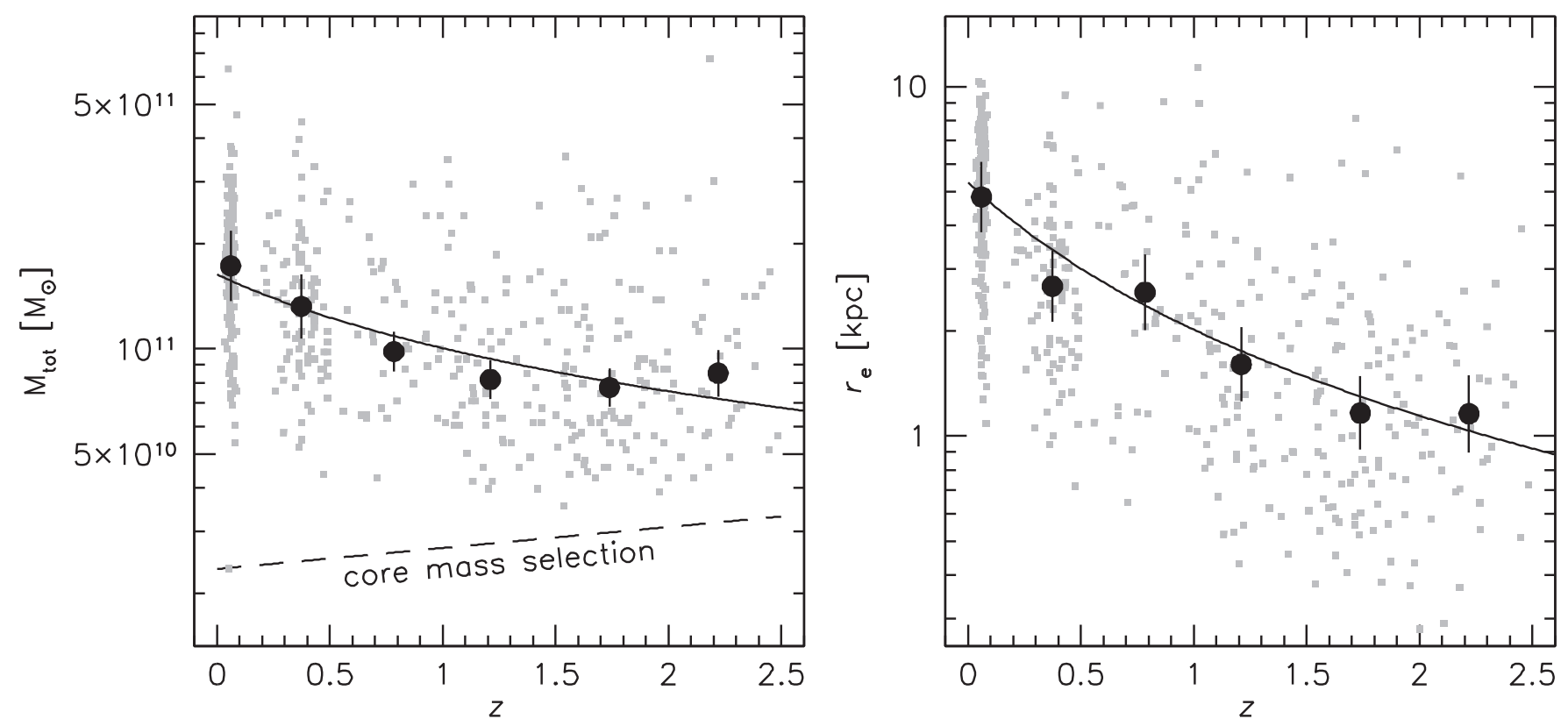

Figure 7. Left panel: total stellar mass of galaxies that have a core mass $\log \left(M_{1 \mathrm{kpc}}\right)=(10.37+0.06 z) \pm 0.05$. Black symbols show the means for SDSS $(z=0.06)$, UltraVISTA/Zürich $(z=0-0.5)$, and 3D-HST $(z=0.5-2.5)$. The mean mass evolves as $M_{\mathrm{tot}} \propto(1+z)^{-0.7}$. Dense cores make up $\sim 50 \%$ of the total mass at $z=2.5$ and $\sim 15 \%$ at $z=0$. Right panel: effective radii of galaxies with dense cores. The effective radius evolves as $r_{e} \propto(1+z)^{-1.4}$.

The evolution of the projected, circularized half-light radius is shown in the right panel of Figure 7. There is strong evolution, such that galaxies with dense cores are more compact at high redshift than at low redshift. The evolution can be described by

$$
\log \left(r_{e}\right)(\mathrm{kpc})=(0.73 \pm 0.06)-(1.40 \pm 0.11) \log (1+z)
$$

The increase in the effective radius is partly caused by adiabatic expansion as a result of mass loss. Using Equation (E1), we estimate that the expansion $\Delta \log \left(r_{e}\right) \sim 0.04 z$, and the corrected evolution is $\log \left(r_{e}\right) \approx 0.64-1.23 \log (1+z)$. The effects of adiabatic expansion are not negligible but small compared to the observed evolution, as previously discussed by Damjanov et al. (2009) in the context of the evolution of the mass-size relation. Note that these corrections assume that mass loss is separable from the processes that cause the increase in the total mass and the effective radius.

We conclude that the average mass and size of galaxies with high core masses evolves with redshift. At high redshift these galaxies are typically compact, with half-light radii of $\sim 1 \mathrm{kpc}$ and about half of the total mass contained in the core. At $z=0$ they are embedded in a large envelope of stars and have effective radii of $\sim 5 \mathrm{kpc}$. These conclusions apply to the population of galaxies that have a massive core, and (as discussed at the beginning of this section) probably also describe the mean evolution of individual galaxies. The cores can then be interpreted as "seeds" around which massive galaxies assemble over time. From $z=2.5$ to $z=0$, the masses of galaxies with dense cores increase by a factor of $\sim 2.4$ and their sizes increase by a factor of $\sim 6$. These results are similar to those derived using number density matching techniques (e.g., van Dokkum et al. 2010; Patel et al. 2013).

\subsection{Stellar Populations}

Galaxies with dense cores have low star formation rates compared to other galaxies with the same total mass and redshift. In the top panels of Figure 8 we show the location of the galaxies with dense cores in the $U V J$ diagram (Labbé et al. 2005; Wuyts et al. 2007; Whitaker et al. 2011). At fixed rest-frame $U-V$ color quiescent galaxies can be separated from dust-reddened star-forming galaxies by their $V-J$ color (see Wuyts et al. 2007). The formal errors in Figure 8 are small, although we note that (1) the colors of galaxies near the extremes of the distribution are influenced by the color range of the EAZY templates, and (2) the location of the boundary between star-forming and quiescent galaxies is somewhat arbitrary. The colors of the galaxies suggest that their rest-frame optical light is dominated by relatively evolved stars, and not by a (reddened) young stellar population. The fraction of quiescent galaxies is $>80 \%$ out to $z=2$ and $57 \%$ at $2<z<2.5$.

The dominance of relatively cool stars in these galaxies is demonstrated directly in the bottom panels of Figure 8, where we show stacked rest-frame spectral energy distributions (SEDs) and WFC3 grism spectra of the galaxies. The spectral stacks were created by de-redshifting the observed data, normalizing in a fixed rest-frame wavelength interval from $4100 \AA$ to $4150 \AA$, and averaging. The SED stacks were created in the same way, except that we plot the individual data points rather than an average in wavelength bins. In all redshift bins the Balmer/ $4000 \AA$ break is clearly detected in the stacked SED and/or the stacked spectrum. The stacked spectra also unambiguously demonstrate that the rest-frame optical emission (and therefore the size and mass measurements) is not greatly influenced by redshift errors or active galactic nucleus (AGN) emission (see also Whitaker et al. 2013).

In Figure 9, we show the correlation between core mass and quiescence, for all galaxies with total masses $>10^{11} M_{\odot}$. The main panel of Figure 9 shows the core mass as a function of redshift, with galaxies color-coded by their location in the $U V J$ diagram. It is striking how well core mass correlates with star formation rate (or, more precisely, with the location in the $U V J$ diagram). The top panel shows the fraction of quiescent and star-forming galaxies as a function of core mass. Essentially all galaxies that lack a dense core are forming stars, and the 


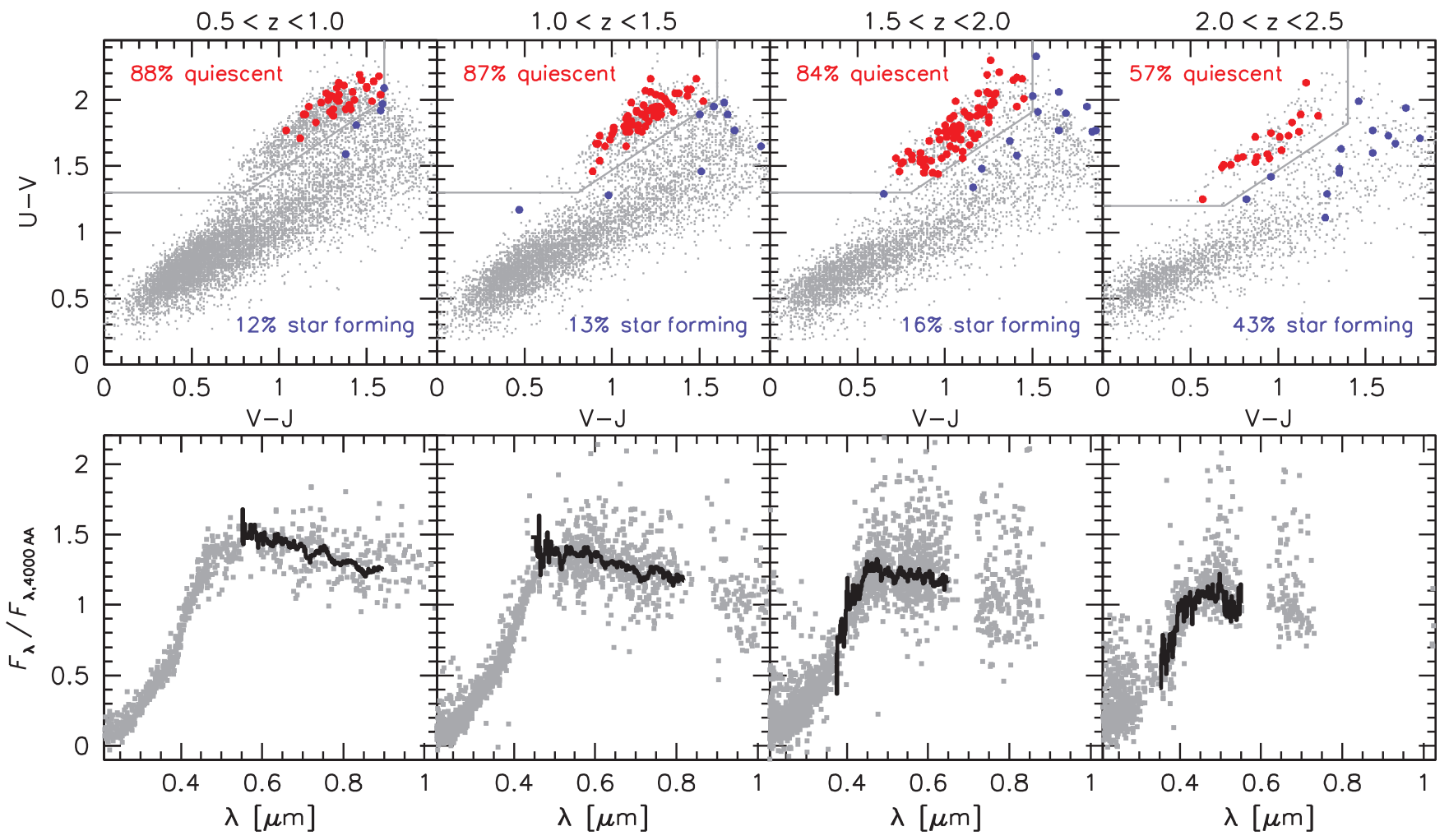

Figure 8. Top panels: location of galaxies with $\log \left(M_{1 \mathrm{kpc}}\right)=(10.37+0.06 z) \pm 0.05$ in the rest-frame $U V J$ plane. Out to $z=2$ more than $80 \%$ of the galaxies have colors indicating evolved stellar populations. The galaxies are among the reddest quiescent galaxies in the local universe. At higher redshifts there is a significant population of star-forming galaxies with dense cores. Bottom panels: stacked SEDs (gray points) and WFC3 grism spectra (black lines) of the galaxies. The galaxies have strong Balmer/4000 A breaks, confirming that their light is dominated by cool stars.

(A color version of this figure is available in the online journal.)

majority of galaxies that have a dense core is quiescent. This result is consistent with the fact that high stellar density is an excellent predictor of quiescence (e.g., Franx et al. 2008).

\subsection{Star-forming Galaxies with Dense Cores}

Although most galaxies with dense cores are quiescent, there are some that fall in the star-forming region of the $U V J$ diagram. Many of these are red in both $U-V$ and $V-J$, indicating significant absorption by dust (Labbé et al. 2005; Wuyts et al. 2007; Marchesini et al. 2014). The fraction of star-forming galaxies with dense cores is as high as $\sim 40 \%$ at $z>2$ (top right panel of Figure 8). This is consistent with many previous studies that have shown that massive star-forming galaxies exist at these redshifts (e.g., Förster Schreiber et al. 2006, 2011; Kriek et al. 2009a; Williams et al. 2010; van Dokkum et al. 2010; Brammer et al. 2011; Marchesini et al. 2014), and with the fact that some of these galaxies have small sizes (e.g., Patel et al. 2013; Barro et al. 2013).

The presence of star-forming galaxies with a high central density does not necessarily mean that we are witnessing the build-up of the dense cores themselves. Significant star formation in the cores would lead to an increase in the core mass, and an increase in the number density of galaxies with dense cores with time-which may be difficult to reconcile with Figures 4 and 5.

Instead, many of these star-forming galaxies could be building mass outside of their centers. As shown in Figure 10, $z>2$ starforming galaxies with dense cores have a median size that is a factor of $\sim$ two larger than that of quiescent galaxies with the same central mass and redshift, which means they are probably not their direct progenitors. Furthermore, for the star-forming galaxies the central $1 \mathrm{kpc}$ contributes only $\sim 25 \%$ of the total mass, which means it is plausible that these galaxies are building up their outer parts rather than their centers. This is qualitatively consistent with other studies of distant galaxies (e.g., Labbé et al. 2003; Nelson et al. 2012, 2013; Wuyts et al. 2013; Genzel et al. 2014) and the properties of spiral galaxies in the local universe.

\subsection{The Fate of Compact Quiescent Galaxies at $z \sim 2$}

The strong mass and size evolution we find for galaxies with dense cores has implications for the evolution of the general population of quiescent galaxies. Many studies have shown that quiescent galaxies with masses of $\sim 10^{11} M_{\odot}$ are very compact at $z \gtrsim 2$, having half-light radii of $\sim 1 \mathrm{kpc}$ (e.g., van Dokkum et al. 2008; Cimatti et al. 2008; van der Wel et al. 2014). Most of these compact quiescent galaxies should have a dense core according to our definition, as galaxies with a projected halflight radius of $\sim 1 \mathrm{kpc}$ have $M_{1 \mathrm{kpc}} \sim 0.5 M_{\mathrm{tot}}$.

The median circularized effective radius of the 31 quiescent galaxies with $10.9<\log M_{\text {tot }}<11.1$ and $2<z<2.5$ is $r_{e}=1.2 \mathrm{kpc}$, consistent with earlier results. The median core mass of these galaxies $\log M_{1 \mathrm{kpc}}=10.6$, with an rms scatter of $0.2 \mathrm{dex}$. Conversely, the median effective radius of galaxies with $10.4<\log M_{1 \mathrm{kpc}}<10.6$ and $2<z<2.5$ is $r_{e}=1.0 \mathrm{kpc}$, their median total mass $\log M_{\mathrm{tot}}=10.9$, and $60 \%$ of these galaxies are quiescent. We conclude that, at $z=2-2.5$, there is substantial overlap between the population of massive quiescent galaxies and the population of galaxies with dense cores (see Figure 2). 


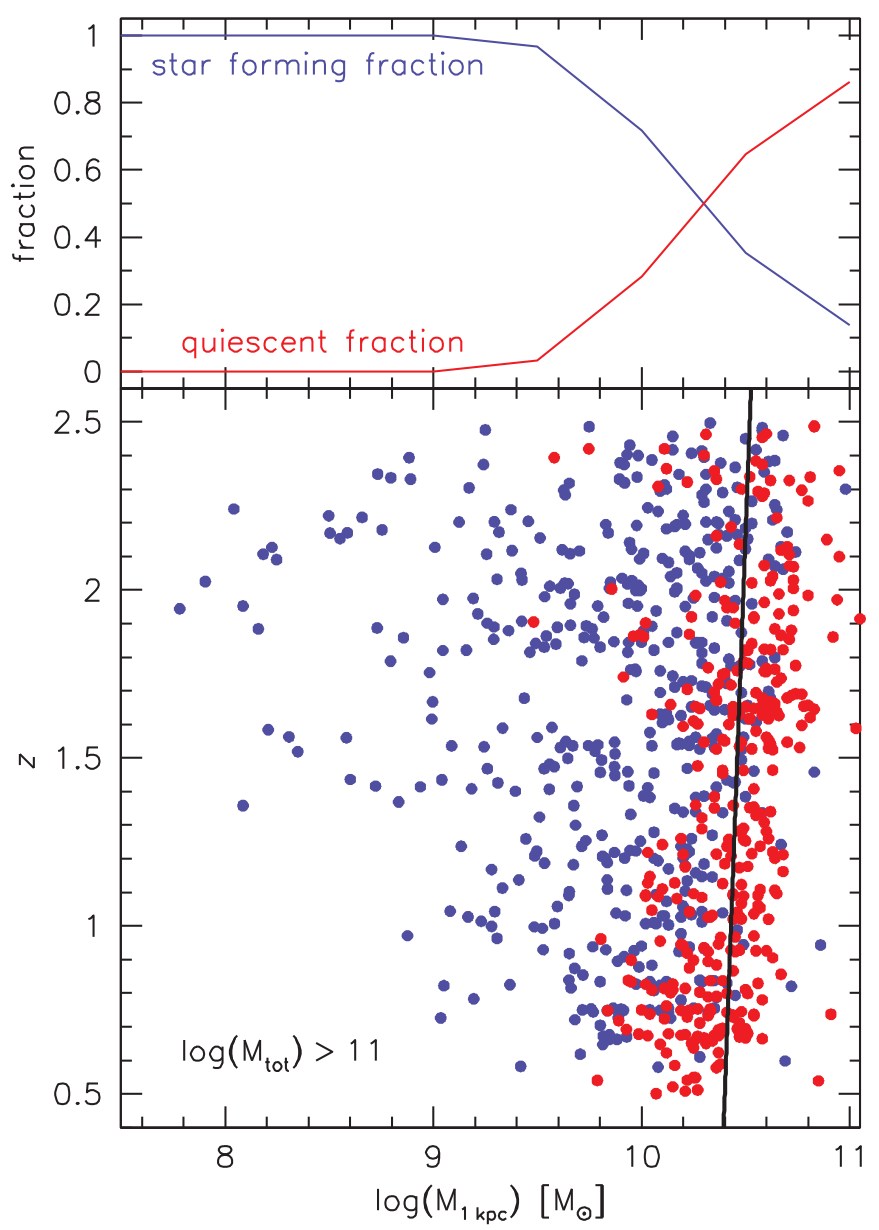

Figure 9. Relation between star formation and core mass, for galaxies with total mass $M_{\text {tot }}>10^{11} M_{\odot}$. Blue points are galaxies that fall in the star-forming part of the $U V J$ diagram; red points are galaxies that fall in the quiescent part. The black line shows our adopted core mass limit (Equation (6)). The top panel shows the fractions of quiescent and star-forming galaxies as a function of core mass. At fixed total mass there is a clear relation between core mass and star formation, and a high core mass seems to be required to stop star formation.

(A color version of this figure is available in the online journal.)

We have shown that (1) the number density of galaxies with dense cores does not increase with time; (2) the half-light radii and total masses of galaxies with dense cores grow with time; and (3) the population of galaxies with dense cores overlaps substantially with the population of massive quiescent galaxies at $z \sim 2$, therefore, compact quiescent galaxies likely grow in size and mass at approximately the same rate as the galaxies with dense cores. This result is consistent with many previous theoretical and observational studies (e.g., Loeb \& Peebles 2003; Bezanson et al. 2009; Naab et al. 2009; Hopkins et al. 2010; van Dokkum et al. 2010; Oser et al. 2010; Trujillo et al. 2011; Newman et al. 2012; Patel et al. 2013; Szomoru et al. 2013; Hilz et al. 2013). It is also consistent with Belli et al. (2014), who made a similar argument based on the structural evolution of galaxies at constant velocity dispersion. It is in conflict with studies that have suggested that massive quiescent galaxies evolve very little since $z \sim 2$ (Carollo et al. 2013; Poggianti et al. 2013), and that the apparent evolution is largely or entirely due to the continuous addition of recently quenched galaxies to the sample. ${ }^{17}$

17 "Progenitor bias"; see van Dokkum \& Franx $(1996,2001)$ for a description of this bias in the context of samples selected by morphology.

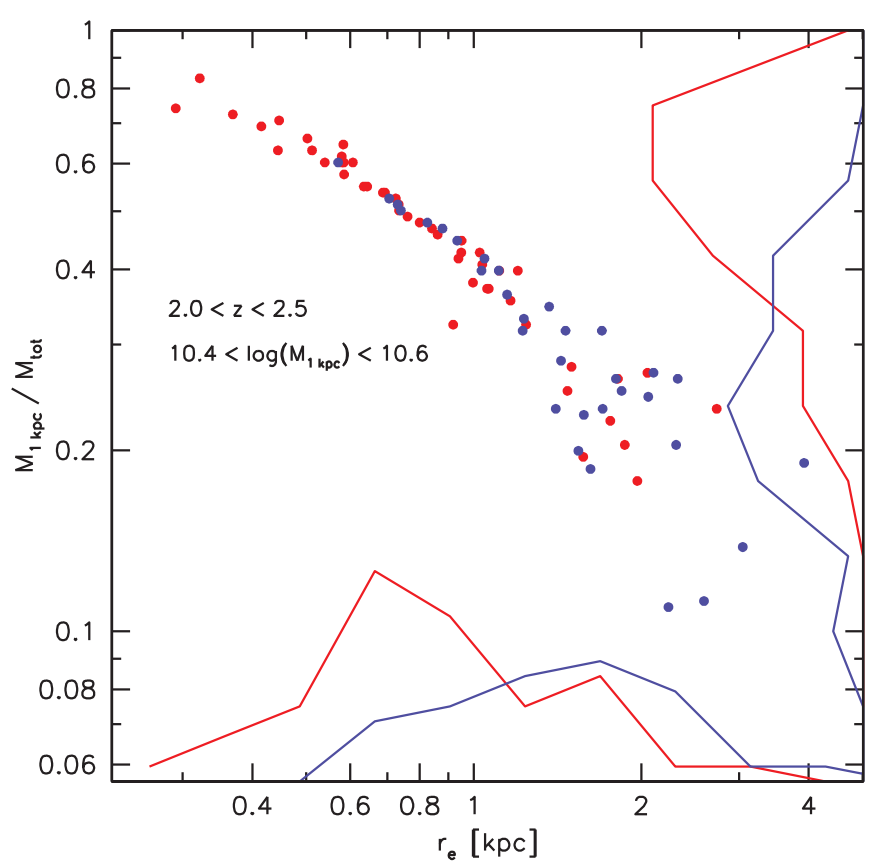

Figure 10. Contribution of the central mass to the total galaxy mass as a function of the projected half-light radius, for $2<z<2.5$ and $\log \left(M_{1 \mathrm{kpc}}\right)=10.5$. Red symbols are quiescent galaxies according to the $U V J$ diagram; blue symbols are star-forming galaxies. Star-forming galaxies are larger than quiescent galaxies at fixed central mass and redshift, and their cores constitute a smaller fraction of the total galaxy mass.

(A color version of this figure is available in the online journal.)

\section{SUMMARY AND CONCLUSIONS}

In this paper, we have identified dense cores in galaxies out to $z=2.5$, using data from the 3D-HST project augmented by low redshift information from UltraVISTA and the SDSS. We find that the evolution of cores with mass $\log \left(M_{1 \mathrm{kpc}}\right) \sim 10.5$ is well described by mild mass loss, suggesting that their stars form a passive stellar population since $z \sim 2.5$. We note that mergers may also contribute to the evolution, and that the effects of mass loss are sensitive to the assumption that $100 \%$ of the stellar ejecta mix with the hot halo gas. At $z \sim 2.5$ the cores make up $\sim 50 \%$ of the total mass of the galaxies that they are part of. At lower redshift they make up a decreasing fraction of the total mass, and by $z=0$ they are embedded in large envelopes of stars with effective radii $\sim 5 \mathrm{kpc}$.

We focused on cores of a fixed high mass, but we note that the evolution of the core mass function is mass dependent (see Figure 3), with low mass cores showing strong positive evolution in their number density. This mass dependence has also been seen in the total mass function (Marchesini et al. 2009) and in the velocity dispersion function (Bezanson et al. 2011). At low masses, star formation may lead to a relatively uniform buildup of galaxies, with the stellar density increasing at all radii, whereas at high masses galaxies are built up inside-out (see van Dokkum et al. 2013).

The negative mass evolution of the cores has consequences for the interpretation of massive star-forming galaxies at $z=$ 1-2.5 and the evolution of quiescent galaxies, as discussed in Section 5. However, we emphasize that not all massive galaxies have dense cores: selecting on total mass produces different samples than selecting on core mass, as is obvious in Figures 2 and 9. Our conclusions only hold for galaxies with a dense core, and leave open the possibility that massive galaxies with 
low core masses have different evolutionary trajectories. It so happens that by $z \sim 2$ our selection mostly overlaps with the population of massive, quiescent galaxies at that redshift, which is why we can rule out several proposed models for their evolution (see Section 5).

We also find that, at fixed total mass and redshift, the presence of a dense core is a good predictor of quiescence and (perhaps more interestingly) its absence is a nearly perfect predictor of star formation (see Figure 9). The latter result is strikingly unambiguous: of 91 galaxies with $M_{\text {tot }}>10^{11} M_{\odot}$ and $M_{1 \mathrm{kpc}}<10^{9.5} M_{\odot}$ only one is quiescent. Apparently the presence of a dense core is a "non-negotiable" requirement for stopping star formation in massive galaxies.

Perhaps the most important result of this paper is that the contribution of stars in dense cores to the stellar mass density of the universe increases strongly with redshift, reaching values of 10\%-20\% at $z \sim 2$ (Section 4.6 and Figure 6(b)). In light of this high fraction, we suggest that the formation of these cores is an important aspect of star formation, galaxy formation, and black hole formation at high redshift.

Interestingly it is not yet clear how this happened. Near the end of their main star formation epoch, prior to stellar mass loss, the cores were even more massive and compact than at $z \sim 2$. The gas mass that was converted to stars inside $1 \mathrm{kpc}$ must have approached $10^{11} M_{\odot}$. Furthermore, this gas must have arrived in the core without forming many stars at larger radii: the quiescent descendants at $z \sim 2$ have small effective radii and no low surface brightness envelopes (e.g., Szomoru et al. 2010, 2013). Several mechanisms have been proposed for creating very compact massive galaxies, such as mergers (Hopkins et al. 2008) and disk instabilities (Dekel \& Burkert 2014). However, reproducing the surface density profiles of the cores has proven to be challenging (see Wuyts et al. 2010). It will also be interesting to see whether models can be created that simultaneously explain the existence of large, massive disks such as that of M101 and of extremely compact cores of similar mass. Forming large disks requires feedback and significant angular momentum (e.g., Guedes et al. 2011), whereas forming dense cores requires rapid cooling and a mechanism to lose angular momentum efficiently (Sales et al. 2012; Dekel \& Burkert 2014). Whatever the mechanism is for getting gas into the center, the core mass will build up quickly when star formation begins. The adiabatic enhancement discussed in Appendix E should also apply "in reverse": when mass is added to the center, the mass within $1 \mathrm{kpc}$ will increase as $\sim\left(M^{\prime} / M\right)^{2}$ due to adiabatic contraction.

Whether forming dense cores have been observed is a matter of debate. As discussed in Section 5 star-forming galaxies with dense cores, such as those identified by Patel et al. (2013) and Barro et al. (2013), may not be forming the core itself but stars away from the center. Spatially resolved star formation maps (e.g., Förster Schreiber et al. 2011; Nelson et al. 2013; Wuyts et al. 2013), or spectroscopy to determine the kinematics of the gas, may provide more information on the location of star formation in these objects. Given the high metallicity of the centers of present-day elliptical galaxies and the high densities, the star-forming cores must have had very large amounts of absorption. They may be largely invisible in the optical and near-IR, and possibly even at larger wavelengths. ${ }^{18}$ Studies of red, far-IR selected galaxies have shed some light on this issue (e.g., Tacconi et al. 2006, 2008; Wang et al. 2012; Gilli et al.

\footnotetext{
18 Somewhat akin to dragonflies, which are aquatic during their nymph stage.
}

2014). The "prototype" would be a dusty star-forming galaxy with a compact morphology and a gas dispersion that matches the dispersion of present-day elliptical galaxies; such an object has recently been identified (Nelson et al. 2014).

The main uncertainty in the analysis is the conversion of light to mass. As discussed in Appendix A and elsewhere, the systematic uncertainties are $\sim 0.1 \mathrm{dex}$, or half of the observed evolution in the core mass. Stellar kinematics are a crucial check on the mass measurements (see, e.g., Bezanson et al. 2013; van de Sande et al. 2013; Belli et al. 2014), although models for the structure of the galaxies and their dark matter are required to interpret them. Furthermore, we have ignored radial gradients in $M / L$ ratio. Our analysis shows that the core masses do not grow but the total masses do, which means the stellar populations in the core are likely different from those at larger radii. The available evidence suggests that these gradients are generally small (Szomoru et al. 2013), but it is difficult to measure them at the relevant spatial scales: $1 \mathrm{kpc}$ corresponds to a single native WFC3 pixel. Spatially resolved studies of strongly lensed galaxies with dense cores could address this issue.

We thank the anonymous referee for constructive comments that improved the manuscript. Support from STScI grant GO12177 is gratefully acknowledged. The UltraVISTA catalog is based on data products from observations made with ESO Telescopes at the La Silla Paranal Observatory under ESO program ID 179.A-2005 and on data products produced by TERAPIX and the Cambridge Astronomy Survey Unit on behalf of the UltraVISTA consortium.

\section{APPENDIX A}

\section{TYING THE WIDE-FIELD COSMOS DATA TO 3D-HST}

In the main text, we augment the 3D-HST survey with data from the UltraVISTA (Muzzin et al. 2013b) and "Zürich" (Sargent et al. 2007) programs in the $1.5 \mathrm{deg}^{2}$ COSMOS field. Here we compare total masses and core masses of objects that are in common between the two surveys, and derive an offset to place the wide-field data on the same system as the 3D-HST data. We also use a third survey, the NMBS (Whitaker et al. 2011). We note that none of these surveys are completely independent; in particular, 3D-HST uses imaging data from both the NMBS and from UltraVISTA.

The comparison sample is limited to objects in the 3D-HST/ CANDELS COSMOS field that have stellar mass measurements from UltraVISTA and GIM2D structural parameters from the Zürich catalog. Figure 11(a) shows the difference in stellar mass between 3D-HST and UltraVISTA, as a function of redshift. Yellow points are objects with $9<\log \left(M_{1 \mathrm{kpc}}\right)<9.8$, and black points are objects with $\log \left(M_{1 \mathrm{kpc}}\right) \geqslant 9.8$. The two surveys produce consistent masses for the vast majority of objects: the median difference for the yellow points is only 0.01. However, the subset of galaxies with high core masses (and the highest total masses) and low redshifts show an offset. The black line is a fit of the form

$\log \left(M_{\text {tot }}\right)($ UltraVISTA $)-\log \left(M_{\text {tot }}\right)(3 \mathrm{D}-\mathrm{HST})=0.23-0.30 z$.

This fit is valid for $\log M_{1 \mathrm{kpc}}>9.8$ and $0.2<z<1$.

In Figure 11(b), we show the difference between masses in 3D-HST and in the NMBS survey (Whitaker et al. 2011). The NMBS is a ground-based $K$-selected survey, like UltraVISTA, 

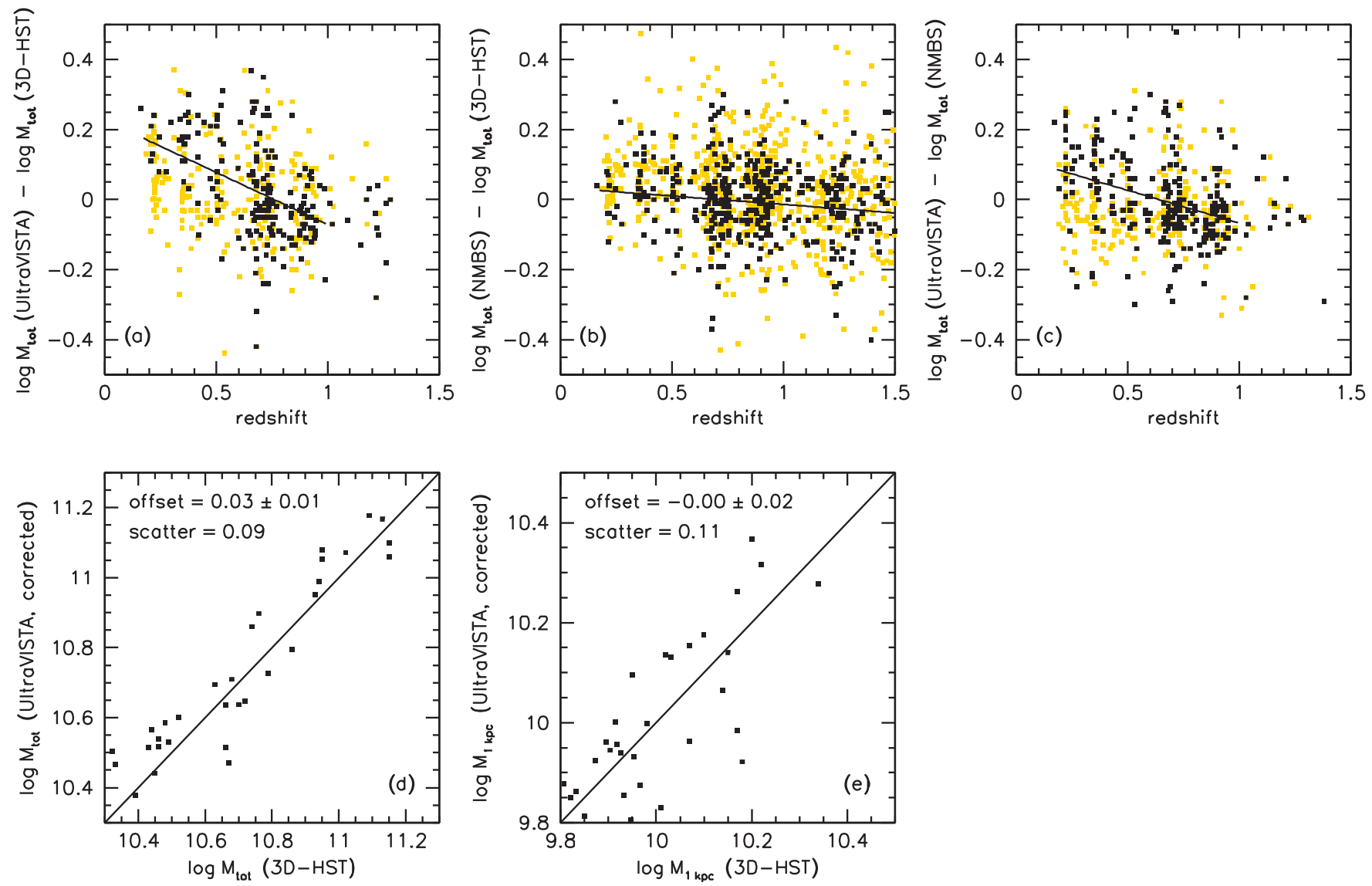

Figure 11. (a) Comparison of total masses measured in UltraVISTA to those measured in 3D-HST, for the same objects. Yellow dots are galaxies with $9<\log \left(M_{1 \mathrm{kpc}}\right)<9.8$; black dots are galaxies with $\log \left(M_{1 \mathrm{kpc}}\right) \geqslant 9.8$. The line is a fit to the black points. (b) Comparison between NMBS and 3D-HST. There is no evidence for a systematic difference. (c) Comparison between UltraVISTA and NMBS. The same difference is evident as between UltraVISTA and 3D-HST: the most massive galaxies at low redshift have slightly higher masses in UltraVISTA. (d) Mass-mass diagram showing total masses in UltraVISTA, offset using the black line in panel (a), vs. 3D-HST masses. (e) Comparison of masses within $1 \mathrm{kpc}$, after offsetting the UltraVISTA masses to the 3D-HST system. The two data sets agree within the uncertainties.

(A color version of this figure is available in the online journal.)

and it uses similar photometric bands. ${ }^{19}$ There is no systematic offset between 3D-HST stellar masses and NMBS stellar masses. This conclusion also applies to the most massive galaxies (black points): the black line is a fit to the most massive objects, and it is within 0.03 dex of zero at all redshifts. Panel (c) compares masses in UltraVISTA to those in NMBS. As expected from panels (a) and (b), we find that UltraVISTA and NMBS have systematically different masses for massive galaxies at low redshift. This subtle redshift-dependent effect is not evident in Figure 8 of Muzzin et al. (2013b); it is present when that figure is remade for the most massive galaxies in the redshift range $0<z<0.5$, at a level consistent with Figure 11(c).

Based on these comparisons, we apply an offset to the UltraVISTA masses, using the relation in Equation (A1). We stress that we do not know whether the masses in UltraVISTA or in 3D-HST are closer to the correct values; we simply adopt the 3D-HST system as our default and add the applied offset in quadrature to our error budget. In Figure 11(d), we show the relation between the total masses in UltraVISTA and in 3D-HST after applying the offset, for galaxies with $\log \left(M_{1 \mathrm{kpc}}\right)>9.8$ and $0<z<0.5$. The difference is now close to zero, as expected. The scatter is 0.09 dex. Assuming that the errors that cause

\footnotetext{
19 The main difference is that NMBS uses medium-bandwidth near-IR filters, which leads to improved photometric redshifts at $z \gtrsim 1$. NMBS covers a $6 \times$ smaller part of the COSMOS field than UltraVISTA.
}

this scatter are of similar magnitude and independent in both surveys (which is an oversimplification), we infer that the error in an individual mass measurement is $0.09 / \sqrt{2}=0.06 \mathrm{dex}$.

In Figure 11(e), we compare the derived core masses for these same galaxies. The core masses were calculated from the total masses and the structural parameters of the galaxies (see Section 3). These structural measurements are completely independent: for 3D-HST they are measured using GALFIT (Peng et al. 2002) from WFC3 $J_{125}$ images (in this redshift range), whereas for COSMOS they were measured using GIM2D (Simard et al. 2002) from ACS $I_{814}$ images. The core masses have an offset of $0.00 \pm 0.02 \mathrm{dex}$, which means the two surveys produce consistent core masses after the offset that we applied to the total masses in UltraVISTA. The random error for a single measurement is approximately 0.08 dex. The core mass function (i.e., the number density of galaxies as a function of their mass within $1 \mathrm{kpc}$ ) in UltraVISTA is shown in Figure 3(b) in the main text; if we had not applied the mass offset the core mass function in this panel would be shifted by +0.09 dex.

As a further check, we compare the core mass function from the wide-field COSMOS data to that derived from 3D-HST. We cannot compare the redshift range $0<z<0.5$ as the 3D-HST data do not sample enough volume (which is why we turned to UltraVISTA/Zürich for this redshift range). However, we can do this comparison at $0.5<z<1$. Here we are constrained by the $I=22.5$ limit of the Zürich morphological catalog; this 


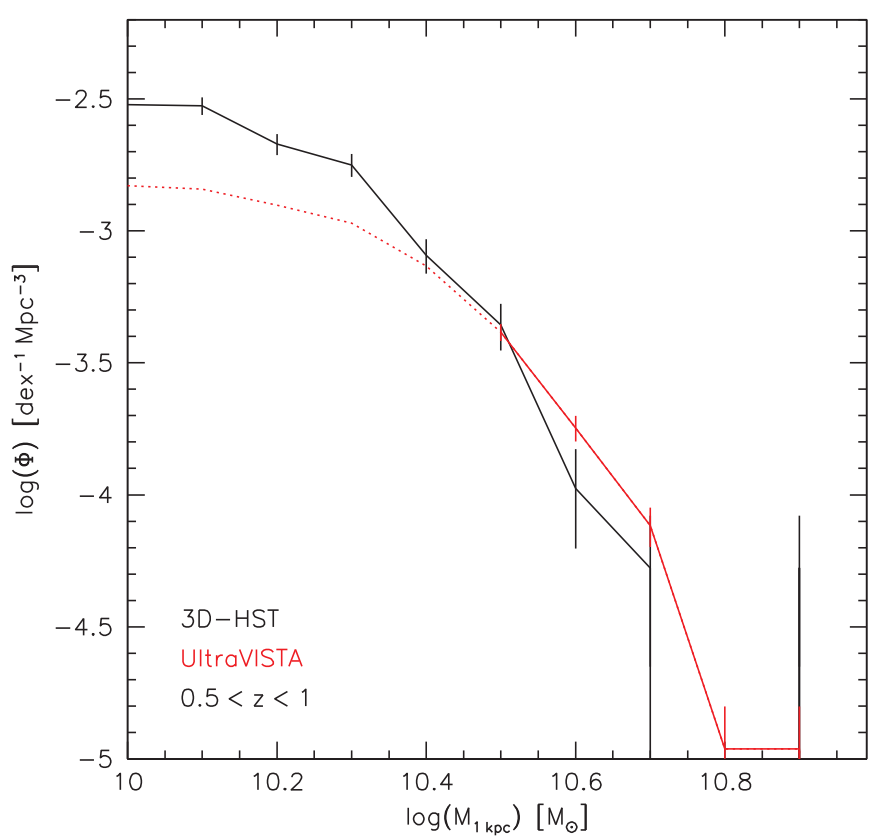

Figure 12. Number density of galaxies as a function of their core mass, for $0.5<z<1$ and data from 3D-HST (black line) and from the UltraVISTA/ Zürich wide-field survey of the COSMOS field (solid red line). The broken red line indicates the regime where the Zürich magnitude limit leads to incompleteness. The two surveys are in good agreement in the regime where they are both complete.

(A color version of this figure is available in the online journal.)

limit implies that the completeness drops below $80 \%$ for core masses $\log \left(M_{1 \mathrm{kpc}}\right) \lesssim 10.5$. We show the comparison between 3D-HST and UltraVISTA/Zürich in Figure 12. The data are in excellent agreement for $\log \left(M_{1 \mathrm{kpc}}\right)>10.4$.

\section{APPENDIX B}

\section{EFFECT OF RANDOM ERRORS ON THE EVOLUTION OF THE CORE MASS FUNCTION}

Due to the steepness of the core mass function, random errors can lead to an artificial increase in the number density of the cores with the highest masses (see, e.g., Bezanson et al. 2011 for an analysis of this effect on the velocity dispersion function). If the random errors are a function of redshift, such that the highest redshift data suffer from the largest errors, they might explain part or all of the observed evolution in the core mass function.

We analyze the effects of random errors in the following way. The observed core masses of individual galaxies in the SDSS are perturbed using a log-normal probability distribution of width $s$. Then, the core mass function is constructed using these perturbed masses and compared to the observed core mass function at $2<z<2.5$. The value of $s$ is related to the errors in the core masses at high redshift, $e_{h}$, and the errors at low redshift, $e_{l}$, through $e_{h}=\left(s^{2}+e_{l}^{2}\right)^{0.5}$.

In Figure 13, we show the effects on the observed core mass function for two values of $s, 0.08$ dex and 0.25 dex. The value of 0.08 is derived from the analysis in Appendix A, where we show that this is the approximate random error $e_{h}$ in an individual measurement in 3D-HST. Assuming that the SDSS measurements have no error and hence $e_{l} \sim 0$, we find $s \sim 0.08$ dex. This can be regarded as a "maximum plausible" error, as there is no a priori reason why the SDSS measurements should have a much smaller error than the high redshift data. It is clear from the dotted line in Figure 13 that random errors

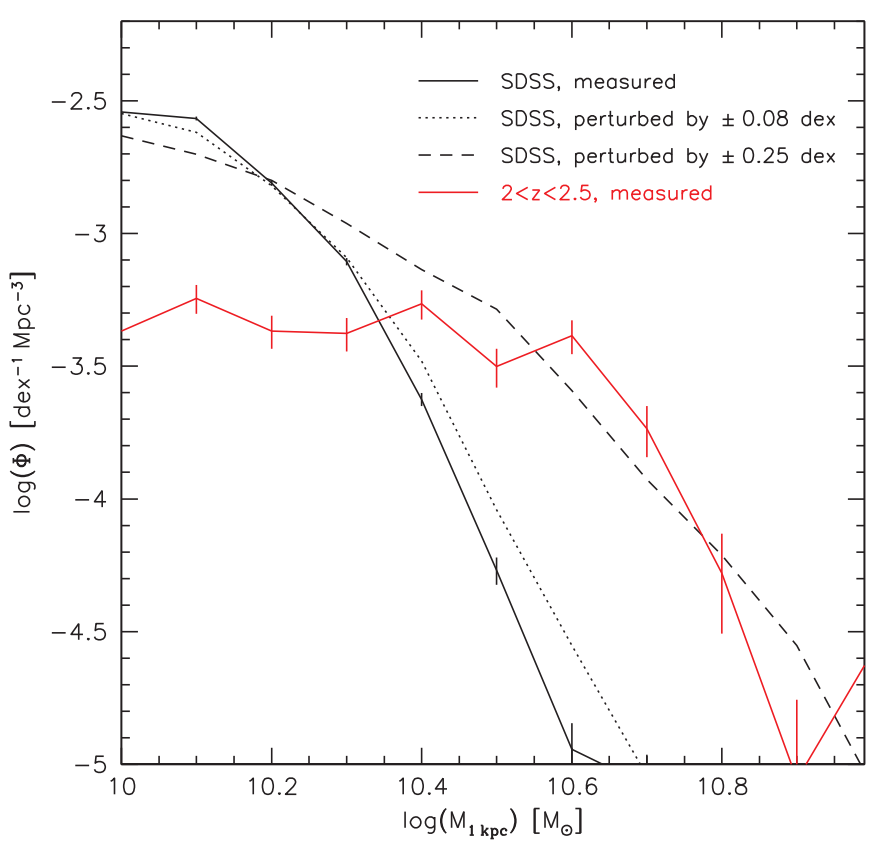

Figure 13. Effect of random errors on the evolution of the core mass function The dotted line shows the SDSS core mass function after perturbing the core masses by a Gaussian of width $s=0.08 \mathrm{dex}$, which is the empirically determined uncertainty in individual measurements in the 3D-HST survey. Random errors in this range cannot explain the observed difference between the core mass function at $2<z<2.5$ and at low redshift. Errors of \pm 0.25 dex (dashed line) would be required to bring the two functions into agreement.

(A color version of this figure is available in the online journal.)

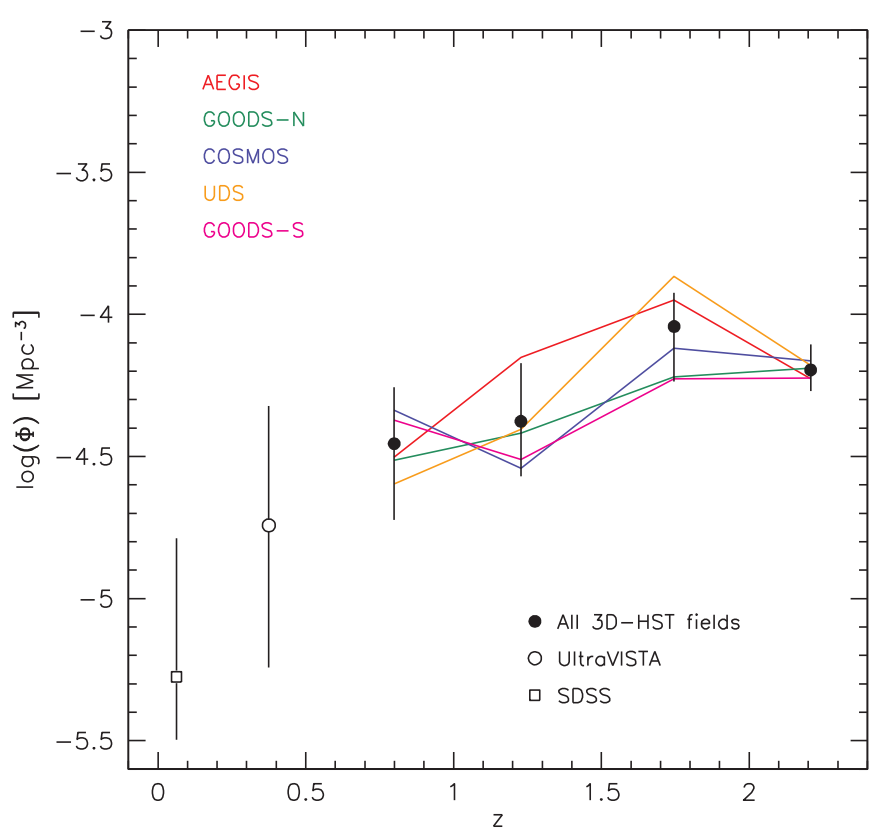

Figure 14. Field-to-field variation in the cumulative number density of dense cores. Black points are identical to those plotted in Figure 4 in the main text. Colored lines show the evolution as measured in each of the five 3D-HST/ CANDELS fields. The evolution is consistent, and the error due to field-to-field variation ("cosmic variance") is small compared to other sources of error.

(A color version of this figure is available in the online journal.)

of this magnitude have little effect on the inferred evolution of the core mass function. To bring the SDSS core mass function into agreement with the observed core mass function at high redshift, the random errors at high redshift would have to be $\sim 0.25$ dex greater than those in the SDSS. 

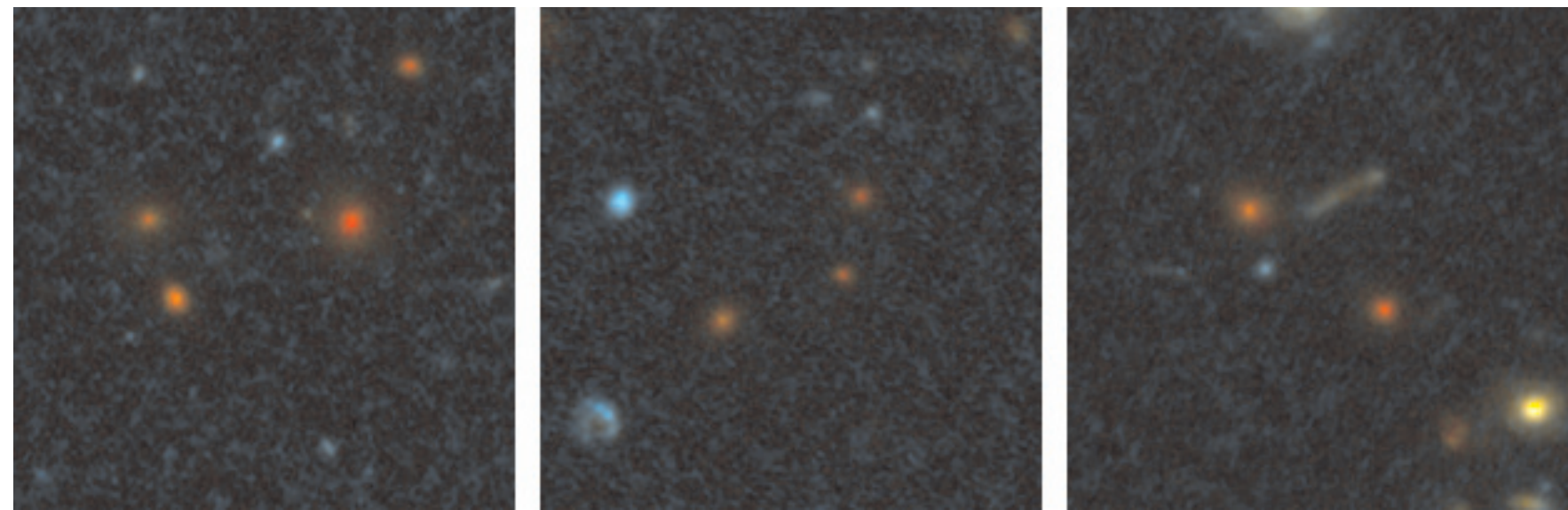

Figure 15. Only pairs of galaxies with dense cores in the entire 3D-HST survey: a pair at $z \approx 1.71$ in the AEGIS field (left), a pair at $z \approx 2.31$ in COSMOS (middle), and a pair at $z \approx 2.07$ in UDS (right). Interestingly, all three pairs have other similarly red galaxies in their vicinity.

(A color version of this figure is available in the online journal.)

\section{APPENDIX C}

\section{FIELD-TO-FIELD VARIATIONS}

Galaxies with dense cores are rare and presumably live in massive dark matter halos; it is therefore a concern that the results in this paper are driven by one or two overdense or underdense regions of the universe. This is a particular concern for the analysis of the evolution of the number density of massive cores (Section 4). Fortunately, the 3D-HST/CANDELS survey covers five survey fields in completely different regions of the sky, and we can test whether the densities in the five fields are similar.

Figure 14 is a repeat of Figure 4, and shows the evolution of the cumulative number density of galaxies with $\log \left(M_{1 \mathrm{kpc}}\right)>$ 10.5. Colored lines show the evolution as measured from the five individual 3D-HST/CANDELS fields. The individual fields show the same evolution as the five fields combined, and there is no single field that significantly alters the average at a particular redshift. The scatter between the fields is $\approx 0.15$ dex for the three lowest redshift bins and only 0.04 dex at $z=2-2.5$. We conclude that the error in the mean due to cosmic variance is approximately $0.15 / \sqrt{5} \approx 0.07 \mathrm{dex}$, much smaller than the errors due to mass uncertainties (see main text). We also infer that there are no large differences in the absolute mass calibrations between the five fields, as they would "translate" into large variation in the normalization of the five curves.

\section{APPENDIX D}

\section{PAIRS OF GALAXIES WITH DENSE CORES}

As discussed in Section 4.3, there are only three pairs of galaxies with dense cores in the 3D-HST/CANDELS fields, out of a parent population of 267 galaxies with $\log \left(M_{1 \mathrm{kpc}}\right)>10.5$. One pair is in the COSMOS field, one in the AEGIS field, and one in the UDS field. No core-core pairs with projected separations $d<43 \mathrm{kpc}$ are found in either of the GOODS fields. Color images of the three pairs are shown in Figure 15.

\section{APPENDIX E}

\section{THE EFFECT OF MASS LOSS ON THE MASS WITHIN $1 \mathrm{kpc}$}

Stellar mass loss can affect the mass within a fixed aperture of $1 \mathrm{kpc}$ in two ways: directly through the mass that is lost in

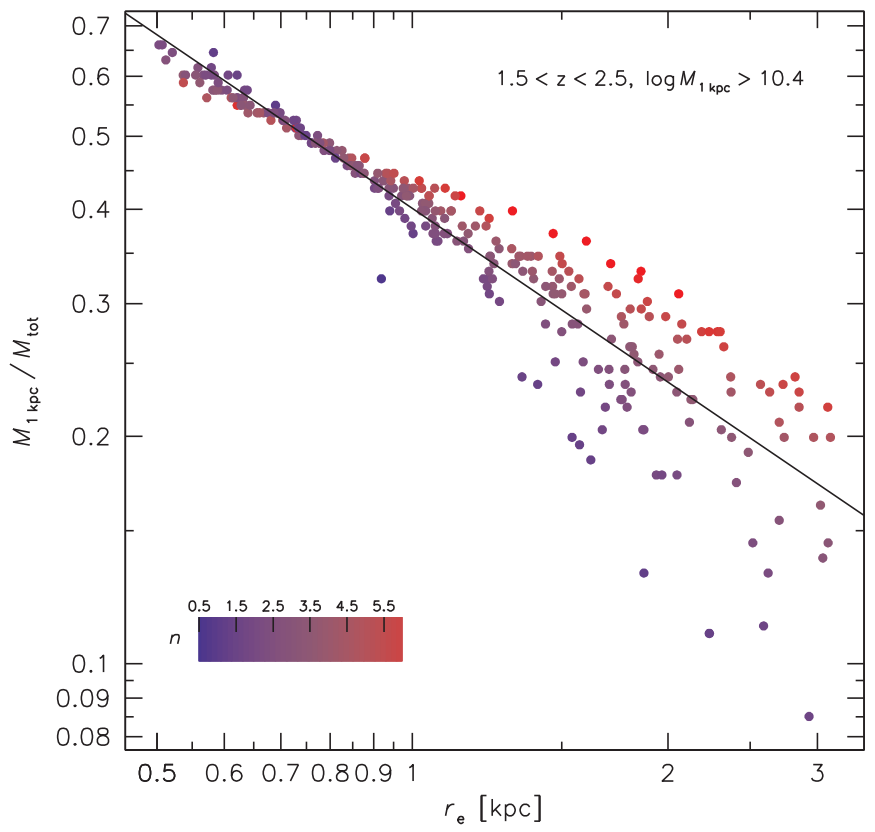

Figure 16. Relation between the effective radius and the fraction of the total mass that is in a dense core, for galaxies with $1.5<z<2.5$ and $\log \left(M_{1 \mathrm{kpc}}\right)>10.5$. Objects are color-coded by their Sersic index, going from blue (low $n$ ) to red (high $n$ ). The line is a fit to all the points, and has a slope of -0.8 . This relation is used to approximate the effect of adiabatic expansion on the mass enclosed within $1 \mathrm{kpc}$.

(A color version of this figure is available in the online journal.)

the winds, and indirectly through the adiabatic expansion that follows the change in mass. As discussed in the main text, the latter effect is only important if $100 \%$ of the stellar ejecta mix with the hot halo gas. Here we calculate the total effect on $M_{1 \mathrm{kpc}}$, the stellar mass within $1 \mathrm{kpc}$, under the assumption that all the material is heated and is diffusely distributed in the hot halo. The galaxy's effective radius will then increase as

$$
r_{e}^{\prime} / r_{e} \sim\left(M^{\prime} / M\right)_{\text {tot }}^{-1}
$$

due to adiabatic expansion (Hills 1980; Fan et al. 2008; RagoneFigueroa \& Granato 2011). The effect on the mass within $1 \mathrm{kpc}$ therefore depends on the structure of the galaxy. If most of the total mass is within $1 \mathrm{kpc}$ to begin with the effect is negligible and $\left(M^{\prime} / M\right)_{1 \mathrm{kpc}} \sim\left(M^{\prime} / M\right)_{\mathrm{tot}}$. On the other hand, if the galaxy 


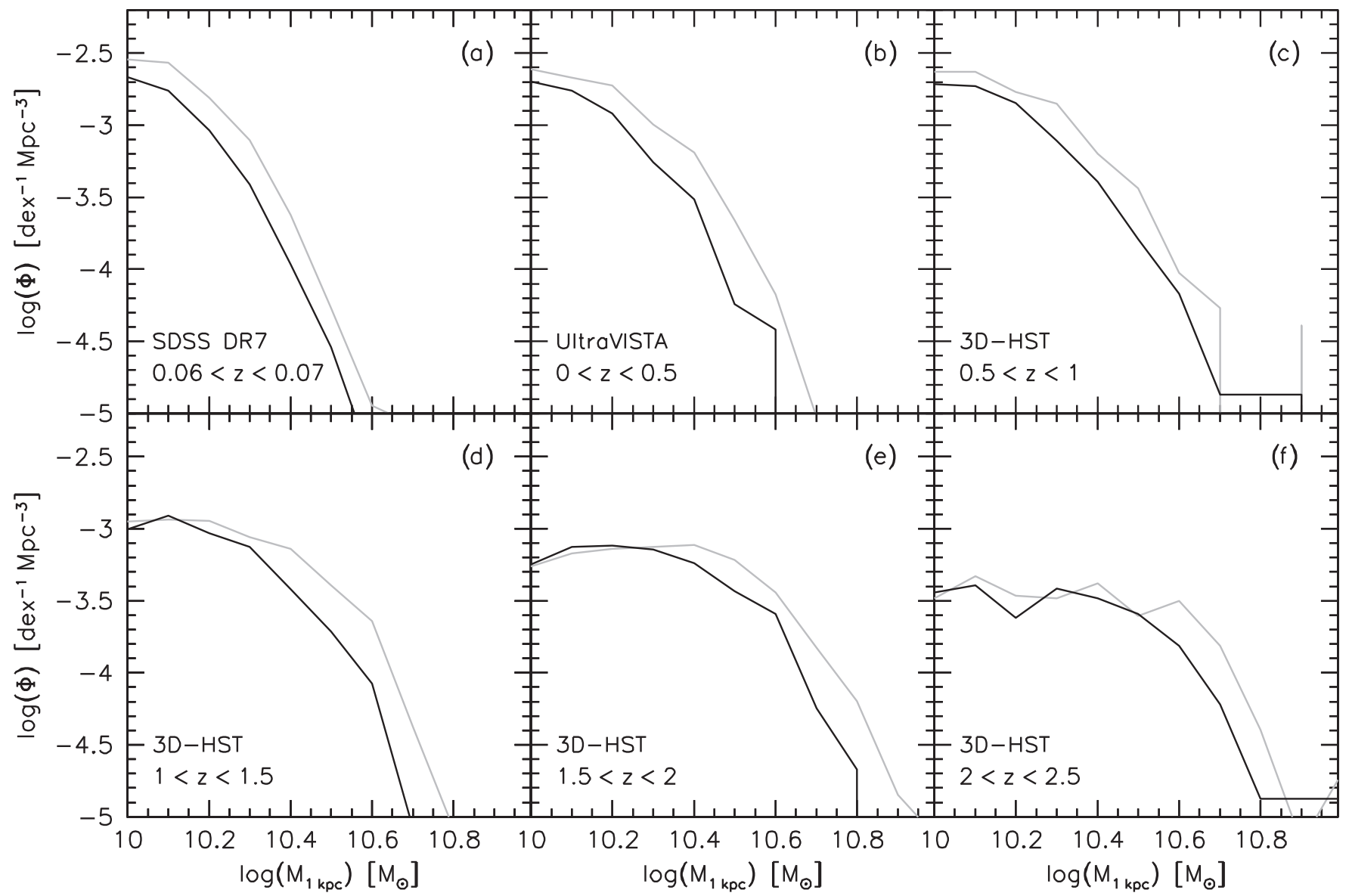

Figure 17. Evolution of the core mass function, using the major axis effective radius in the deprojection rather than the circularized effective radius (black lines). The default deprojections used in the paper are shown in gray. There is a constant offset in mass, as expected, but no significant redshift-dependent difference between the grey and black curves.

has a density profile that is nearly constant with radius (and therefore $\left.r_{e} \gg 1 \mathrm{kpc}\right)$, the mass inside $1 \mathrm{kpc}$ will decrease as $\left(M^{\prime} / M\right)_{1 \mathrm{kpc}} \sim\left(M^{\prime} / M\right)_{\mathrm{tot}} \times\left(r_{e}^{\prime} / r_{e}\right)^{-3} \sim\left(M^{\prime} / M\right)_{\mathrm{tot}}^{4}$

In practice, the effect on $M_{1 \mathrm{kpc}}$ will be in between these two extremes. We determined the change in $M_{1 \mathrm{kpc}}$ empirically using the actual galaxies in the 3D-HST survey. Figure 16 shows the ratio between $M_{1 \mathrm{kpc}}$ and $M_{\mathrm{tot}}$ as a function of the effective radius, for galaxies with $\log \left(M_{1 \mathrm{kpc}}\right)>10.4,1.5<z<2.5$, and $-0.3<\log \left(r_{e}\right)<0.5$. The slope of the relation ranges between -0.6 and -1.3 depending on the Sersic (1968) index. The average relation, shown by the solid line, is $M_{1 \mathrm{kpc}} / M_{\mathrm{tot}} \propto r_{e}^{-0.8}$.

We infer that the total effect of stellar mass loss on the mass within $1 \mathrm{kpc}$ is

$$
\begin{aligned}
\left(M^{\prime} / M\right)_{1 \mathrm{kpc}} & \sim\left(M^{\prime} / M\right)_{\mathrm{tot}} \times\left(r_{e}^{\prime} / r_{e}\right)^{-0.8} \\
& \sim\left(M^{\prime} / M\right)_{\mathrm{tot}}^{1.8} .
\end{aligned}
$$

We note that this relation assumes that there are no other sources of mass loss than stellar evolution. If there are other sources of mass loss, such as AGN-driven winds (e.g., Fan et al. 2008), the adiabatic component of Equation (E2) may be larger.

\section{APPENDIX F}

\section{CORE MASS FUNCTION USING MAJOR AXIS EFFECTIVE RADII}

In the default analysis in the paper, we use the circularized effective radius to deproject the best-fitting Sersic (1968) profiles and measure the core mass. As noted in Section 4.5, the circularization may introduce biases if the mean axis ratio of galaxies evolves with redshift: at fixed circularized effective radius, highly flattened galaxies have less mass in a sphere of $1 \mathrm{kpc}$ than spherical galaxies. We determined the importance of this effect by repeating the deprojection, now using the major axis effective radius rather than the circularized effective radius. The major axis effective radius is always larger than the circularized one, and so the inferred core masses decrease; the question is whether this decrease is dependent on redshift.

The core mass function, as derived using the major axis effective radius, is shown by the black lines in Figure 17. It is compared to the original, circularized measurements in grey. There is no significant redshift-dependent effect, and we conclude that systematic changes in axis ratio are not the cause of the apparent negative evolution in the number density of dense cores.

\section{REFERENCES}

Barro, G., Faber, S. M., Pérez-González, P. G., et al. 2013, ApJ, 765, 104 Begelman, M. C., Blandford, R. D., \& Rees, M. J. 1980, Natur, 287, 307 Bell, E. F., Naab, T., McIntosh, D. H., et al. 2006, ApJ, 640, 241

Bell, E. F., van der Wel, A., Papovich, C., et al. 2012, ApJ, 753, 167 Belli, S., Newman, A. B., \& Ellis, R. S. 2014, ApJL, 788, L29

Bezanson, R., van Dokkum, P. G., Franx, M., et al. 2011, ApJL, 737, L31 Bezanson, R., van Dokkum, P. G., Tal, T., et al. 2009, ApJ, 697, 1290 Bezanson, R., van Dokkum, P. G., van de Sande, J., et al. 2013, ApJL, 779, L21 Bluck, A. F. L., Conselice, C. J., Buitrago, F., et al. 2012, ApJ, 747, 34 Brammer, G. B., van Dokkum, P. G., \& Coppi, P. 2008, ApJ, 686, 1503 
Brammer, G. B., van Dokkum, P. G., Franx, M., et al. 2012, ApJS, 200, 13 Brammer, G. B., van Dokkum, P. G., Illingworth, G. D., et al. 2013, ApJL, 765, L2

Brammer, G. B., Whitaker, K. E., van Dokkum, P. G., et al. 2011, ApJ, 739, 24

Bregman, J. N., \& Parriott, J. R. 2009, ApJ, 699, 923

Brinchmann, J., Charlot, S., White, S. D. M., et al. 2004, MNRAS, 351, 1151

Bruzual, G., \& Charlot, S. 2003, MNRAS, 344, 1000

Bundy, K., Fukugita, M., Ellis, R. S., et al. 2009, ApJ, 697, 1369

Cappellari, M., McDermid, R. M., Alatalo, K., et al. 2012, Natur, 484, 485

Carollo, C. M., Bschorr, T. J., Renzini, A., et al. 2013, ApJ, 773, 112

Cassata, P., Giavalisco, M., Williams, C. C., et al. 2013, ApJ, 775, 106

Chabrier, G. 2003, PASP, 115, 763

Cimatti, A., Cassata, P., Pozzetti, L., et al. 2008, A\&A, 482, 21

Conroy, C., \& van Dokkum, P. G. 2012, ApJ, 760, 71

Conroy, C., van Dokkum, P. G., \& Kravtsov, A. 2014, ApJL, submitted (arXiv:1406.3026)

Daddi, E., Renzini, A., Pirzkal, N., et al. 2005, ApJ, 626, 680

Damjanov, I., McCarthy, P. J., Abraham, R. G., et al. 2009, ApJ, 695, 101

Davies, R. L., Sadler, E. M., \& Peletier, R. F. 1993, MNRAS, 262, 650

Dekel, A., \& Burkert, A. 2014, MNRAS, 438, 1870

Dickinson, M., Papovich, C., Ferguson, H. C., \& Budavári, T. 2003, ApJ, 587,25

El-Zant, A. A., Kim, W.-T., \& Kamionkowski, M. 2004, MNRAS, 354, 169

Faber, S. M., Tremaine, S., Ajhar, E. A., et al. 1997, AJ, 114, 1771

Fan, L., Lapi, A., De Zotti, G., \& Danese, L. 2008, ApJL, 689, L101

Feldmann, R., Carollo, C. M., Mayer, L., et al. 2010, ApJ, 709, 218

Ferrarese, L., \& Merritt, D. 2000, ApJL, 539, L9

Förster Schreiber, N. M., Genzel, R., Lehnert, M. D., et al. 2006, ApJ, 645, 1062

Förster Schreiber, N. M., Shapley, A. E., Erb, D. K., et al. 2011, ApJ, 731, 65

Franx, M., \& Illingworth, G. 1990, ApJL, 359, L41

Franx, M., van Dokkum, P. G., Schreiber, N. M. F., et al. 2008, ApJ, 688, 770

Gebhardt, K., Bender, R., Bower, G., et al. 2000, ApJL, 539, L13

Genzel, R., Förster Schreiber, N. M., Lang, P., et al. 2014, ApJ, 785, 75

Gilli, R., Norman, C., Vignali, C., et al. 2014, A\&A, 562, A67

Grogin, N. A., Kocevski, D. D., Faber, S. M., et al. 2011, ApJS, 197, 35

Guedes, J., Callegari, S., Madau, P., \& Mayer, L. 2011, ApJ, 742, 76

Gültekin, K., Richstone, D. O., Gebhardt, K., et al. 2009, ApJ, 698, 198

Hills, J. G. 1980, ApJ, 235, 986

Hilz, M., Naab, T., \& Ostriker, J. P. 2013, MNRAS, 429, 2924

Hopkins, P. F. 2013, MNRAS, 433, 170

Hopkins, P. F., Bundy, K., Hernquist, L., Wuyts, S., \& Cox, T. J. 2010, MNRAS, 401, 1099

Hopkins, P. F., Cox, T. J., Kereš, D., \& Hernquist, L. 2008, ApJS, 175, 390

Hopkins, P. F., \& Hernquist, L. 2010, MNRAS, 407, 447

Kauffmann, G., Heckman, T. M., White, S. D. M., et al. 2003, MNRAS, 341,54

Kitzbichler, M. G., \& White, S. D. M. 2008, MNRAS, 391, 1489

Koekemoer, A. M., Faber, S. M., Ferguson, H. C., et al. 2011, ApJS, 197, 36

Kormendy, J., \& Bender, R. 2009, ApJL, 691, L142

Kormendy, J., \& Sanders, D. B. 1992, ApJL, 390, L53

Kriek, M., van Dokkum, P. G., Franx, M., Illingworth, G. D., \& Magee, D. K. 2009a, ApJL, 705, L71

Kriek, M., van Dokkum, P. G., Labbé, I., et al. 2009b, ApJ, 700, 221

Kroupa, P. 2001, MNRAS, 322, 231

Krumholz, M. R. 2011, ApJ, 743, 110

Kuntschner, H., Emsellem, E., Bacon, R., et al. 2010, MNRAS, 408, 97

Kuntschner, H., Lucey, J. R., Smith, R. J., Hudson, M. J., \& Davies, R. L. 2001, MNRAS, 323, 615

Labbé, I., Huang, J., Franx, M., et al. 2005, ApJL, 624, L81

Labbé, I., Rudnick, G., Franx, M., et al. 2003, ApJL, 591, L95

Leja, J., van Dokkum, P., \& Franx, M. 2013, ApJ, 766, 33

Loeb, A., \& Peebles, P. J. E. 2003, ApJ, 589, 29

Magorrian, J., Tremaine, S., Richstone, D., et al. 1998, AJ, 115, 2285

Marchesini, D., Muzzin, A., Stefanon, M., et al. 2014, arXiv:1402.0003

Marchesini, D., van Dokkum, P. G., Förster Schreiber, N. M., et al. 2009, ApJ, 701,1765
McCracken, H. J., Milvang-Jensen, B., Dunlop, J., et al. 2012, A\&A, 544, 156 Milosavljević, M., \& Merritt, D. 2001, ApJ, 563, 34

Muzzin, A., Marchesini, D., Stefanon, M., et al. 2013a, ApJ, 777, 18

Muzzin, A., Marchesini, D., Stefanon, M., et al. 2013b, ApJS, 206, 8

Naab, T., Johansson, P. H., \& Ostriker, J. P. 2009, ApJL, 699, L178

Nelson, E., van Dokkum, P., Franx, M., et al. 2014, Natur, in press (arXiv:1406.3350)

Nelson, E. J., van Dokkum, P. G., Brammer, G., et al. 2012, ApJL, 747, L28

Nelson, E. J., van Dokkum, P. G., Momcheva, I., et al. 2013, ApJL, 763, L16

Newman, A. B., Ellis, R. S., Bundy, K., \& Treu, T. 2012, ApJ, 746, 162

Oser, L., Naab, T., Ostriker, J. P., \& Johansson, P. H. 2012, ApJ, 744, 63

Oser, L., Ostriker, J. P., Naab, T., Johansson, P. H., \& Burkert, A. 2010, ApJ, 725, 2312

Patel, S. G., van Dokkum, P. G., Franx, M., et al. 2013, ApJ, 766, 15

Peng, C. Y., Ho, L. C., Impey, C. D., \& Rix, H.-W. 2002, AJ, 124, 266

Poggianti, B. M., Calvi, R., Bindoni, D., et al. 2013, ApJ, 762, 77

Ragone-Figueroa, C., \& Granato, G. L. 2011, MNRAS, 414, 3690

Rudnick, G., Rix, H., Franx, M., et al. 2003, ApJ, 599, 847

Sales, L. V., Navarro, J. F., Theuns, T., et al. 2012, MNRAS, 423, 1544

Salpeter, E. E. 1955, ApJ, 121, 161

Sargent, M. T., Carollo, C. M., Lilly, S. J., et al. 2007, ApJS, 172, 434

Scoville, N., Aussel, H., Brusa, M., et al. 2007, ApJS, 172, 1

Sersic, J. L. 1968, Atlas de Galaxias Australes (Cordoba, Argentina: Observatorio Astronomico)

Simard, L., Mendel, J. T., Patton, D. R., Ellison, S. L., \& McConnachie, A. W. 2011, ApJS, 196, 11

Simard, L., Willmer, C. N. A., Vogt, N. P., et al. 2002, ApJS, 142, 1

Skelton, R. E., Whitaker, K. E., Momcheva, I. G., et al. 2014, ApJS, submitted (arXiv:1403.3689)

Solomon, P. M., Downes, D., \& Radford, S. J. E. 1992, ApJL, 387, L55

Szomoru, D., Franx, M., van Dokkum, P. G., et al. 2010, ApJL, 714, L244

Szomoru, D., Franx, M., van Dokkum, P. G., et al. 2013, ApJ, 763, 73

Tacconi, L. J., Genzel, R., Smail, I., et al. 2008, ApJ, 680, 246

Tacconi, L. J., Neri, R., Chapman, S. C., et al. 2006, ApJ, 640, 228

Taylor, E. N., Franx, M., Brinchmann, J., van der Wel, A., \& van Dokkum, P. G. 2010a, ApJ, 722, 1

Taylor, E. N., Franx, M., Glazebrook, K., et al. 2010b, ApJ, 720, 723

Treu, T., Auger, M. W., Koopmans, L. V. E., et al. 2010, ApJ, 709, 1195

Trujillo, I., Cenarro, A. J., de Lorenzo-Cáceres, A., et al. 2009, ApJL, 692, L118

Trujillo, I., Ferreras, I., \& de La Rosa, I. G. 2011, MNRAS, 415, 3903

Trujillo, I., Förster Schreiber, N. M., Rudnick, G., et al. 2006, ApJ, 650, 18

van de Sande, J., Kriek, M., Franx, M., et al. 2013, ApJ, 771, 85

van der Wel, A., Bell, E. F., Häussler, B., et al. 2012, ApJS, 203, 24

van der Wel, A., Bell, E. F., van den Bosch, F. C., Gallazzi, A., \& Rix, H.-W. 2009, ApJ, 698, 1232

van der Wel, A., Franx, M., van Dokkum, P. G., et al. 2014, ApJ, 788, 28

van Dokkum, P. G. 2005, AJ, 130, 2647

van Dokkum, P. G., \& Conroy, C. 2010, Natur, 468, 940

van Dokkum, P. G., \& Franx, M. 1996, MNRAS, 281, 985

van Dokkum, P. G., \& Franx, M. 2001, ApJ, 553, 90

van Dokkum, P. G., Franx, M., Kriek, M., et al. 2008, ApJL, 677, L5

van Dokkum, P. G., Leja, J., Nelson, E. J., et al. 2013, ApJL, 771, L35

van Dokkum, P. G., Whitaker, K. E., Brammer, G., et al. 2010, ApJ, 709, 1018

Wake, D. A., van Dokkum, P. G., \& Franx, M. 2012, ApJL, 751, L44

Wang, W.-H., Barger, A. J., \& Cowie, L. L. 2012, ApJ, 744, 155

Weinmann, S. M., Franx, M., van Dokkum, P., \& Bezanson, R. 2013, ApJL, 767, L21

Whitaker, K. E., Labbé, I., van Dokkum, P. G., et al. 2011, ApJ, 735, 86

Whitaker, K. E., van Dokkum, P. G., Brammer, G., et al. 2013, ApJL, 770, L39

Williams, R. J., Quadri, R. F., \& Franx, M. 2011, ApJL, 738, L25

Williams, R. J., Quadri, R. F., Franx, M., et al. 2010, ApJ, 713, 738

Worthey, G., Faber, S. M., \& Gonzalez, J. J. 1992, ApJ, 398, 69

Wuyts, S., Cox, T. J., Hayward, C. C., et al. 2010, ApJ, 722, 1666

Wuyts, S., Förster Schreiber, N. M., Nelson, E. J., et al. 2013, ApJ, 779, 135

Wuyts, S., Labbé, I., Franx, M., et al. 2007, ApJ, 655, 51 